\title{
EVALUATION OF THE GENETIC VARIATION FOR SOME GENOTYPES IN COTTON (GOSSYPIUM BARBADENSE L.) TO WATER STRESS
}

\author{
M.A.A. El-Dahan, A.B.A. El-Fesheikawy and Y.I.M. AL-Hibbiny \\ Cotton Research Institute, Agricultural Research Center, Giza, Egypt \\ Received: Jan. 30,2018 \\ Accepted: Mar. 1, 2018
}

ABSTRACT: Sixteen cotton genotypes representing a wide range of cotton characteristics, were used in two concurrent trials (laboratory and field) at Sakha Agric. Res. Station under well-watered and water-limited regimes during 2016 and 2017 seasons. The objectives were to determine genotypic variability among cotton varieties in their response to deficit water stress and to detect the most suitable genotypes, traits and selection procedure for water stress condition. The genotypes were evaluated for water deficit stress in laboratory by the simulation of water potentials with polyethylene glycol-6000 (0.0 and -0.4 MPa), at 25ㄷ using aerated hydroponic culture box. After 18 days the following seedling traits were measured, root length $(\mathrm{cm})(R L)$, root fresh weight (g) (RFW), root dry weight (g) (RDW), shoot length (cm) (SHL), shoot fresh weight (g) (SHFW) and shoot dry weight (g) (SHDW). Field trail conducted to measure vegetative traits, yield and yield components and fiber traits. Analysis of variance for the growing seasons 2016 and 2017 revealed significant differences with respect to water regimes, genotypes and water regimes $x$ genotypes for most of the studied traits, confirming the presence of genotypic variability among the studied cotton genotypes. Mean values exhibited decreasing in traits from normal to water deficit conditions in all traits except for maturity (M). The relative reduction (RD\%) varied from $1.25 \%$ for pressley index $(\mathrm{PI})$ to $51.6 \%$ for lint yield/plant (LY/P). Fiber traits were the lowest affected traits by water stress. G. 88, G. 93 and Suvin were less affected by water stress for seedling traits. For vegetative traits, G.77, G.94, G.89xG.86, Ashmouni, Menoufi and Suvin showed higher values under water deficit. Regarding to yield and yield components traits G.89xG.86, Menoufi, Suvin and G.86 showed the highest water deficit tolerance with acceptable production under limited water regime. On the other hand, most of extra-long staple cotton varieties G.87, G.88 and G.93 were most susceptible to water deficit stress in production term. Drought susceptibility index (DSI) showed significant negative correlation with yield under water deficit regime suggesting DSI as a useful predictor of drought tolerance in cotton and confirming the need of performing genotype evaluation under water stress in case of breeding for water deficit tolerance. Generally, the extralong genotypes were more susceptible to water deficit stress than long staple genotypes for fiber traits. Correlation coefficients between all the studied traits under well watered and limited water regimes over two years revealed that, yield was positively correlated with yield components traits and plant height (PH); and negatively correlated with most fiber traits. The path coefficient analysis revealed positive and negative direct effect of traits on seed cotton yield (SCY/P). The highest direct effect on SCY/P was exhibited by bolls/plant $(B / P)(1.36)$ followed by boll weight $(B W)(0.91)$ and lint percentage $(L \%)(0.53)$. The highest indirect effect of most of yield and vegetative traits were through $B / P$ and $B W$. These results confirming that, selection to improve productivity under water deficit stress could be more effective throughout direct selection for B/P and BW. Factor analysis revealed that first three components accounted for about $88.51 \% \%$ of the total variation among the studied traits. Results exhibited the importance of $L I, S I, L \%, B W$, $L Y / P, S C Y / P$ and $P H$ traits in factor 1 and $B / P, S C Y / P$ and $L Y / P$ in factor 2 confirming the 
M.A.A. El-Dahan, et al.,

importance of these traits in the total variance to improve productivity under deficit water stress.

Key words: Genotypic Variation, Drought susceptibility index, Cotton.

\section{INTRODUCTION}

Water deficit is the major abiotic stress factor limiting plant growth and crop productivity around the world (Kramer, 1983; Turner, 1997; Sinclair, 2005). Approximately one third of the cultivated area of the world suffers from inadequate supplies of water (Massacci et al., 2008). In all agricultural regions, yields of rain-fed crops are periodically reduced by drought (Kramer, 1983), and the severity of the problem may increase due to changing world climatic trends (Le Houerou, 1996). In general, plant water stress is defined as the condition where a plant's water potential and turgor are decreased enough to inhibit normal plant function (Hsiao et al., 1976). The effects of water stress depend on the severity and duration of the stress, the growth stage at which stress is imposed, and the genotype of the plant (Kramer, 1983).

Many studies have reported how cotton reproductive growth, yield and fiber quality are affected by water deficits. Cotton yield is dependent on the production and retention of bolls, and both can be decreased by water stress (Guinn and Mauney, 1984). Under water stress, decrease in seed cotton yield is primarily due to the reduction in number of bolls and boll weight (Pettigrew, 2004 b; Wang et al., 2004; Mert, 2005; Basal et al., 2009). Water stress affects lint quality in numerous ways, especially during the fiber elongation period, which results in a decrease in fiber length and causes fiber immaturity (Ritchie et al., 2004 ; Mert, 2005).

Many studies showed that there is genotypic variability for water-deficit stress in cotton (Quisenberry et al., 1981; Lacape et al., 1998; Pettigrew and
Meredith, 1994). Therefore, selection for drought tolerance is a major interest of plant breeders in cotton. A number of different morphological (leaf, stem and root growth parameters) and physiological traits (more than $\mathbf{3 0}$ traits) have been suggested as important selection criteria relative to drought tolerance in cotton (Loka et al., 2011). However, none of these physiological traits has so far been consistently correlated positively with drought tolerance (Loka et al., 2011). The difficulty in identification of a physiological parameter as a reliable indicator of yield in drought conditions has suggested that yield performance over a range of environments should be used as the main indicator for drought tolerance (Voltas et al., 2005).

One of the most commonly methods used to determine the tolerance of plants to abiotic stresses is the evaluation of the germination capacity of seeds under such conditions (Larcher, 2000). Aiming to simulate water stress conditions in the laboratory, germination studies have been carried out with aqueous solutions of polyethyleneglycol-6000 (PEG-6000) and mannitol (Murillo-Amador et al., 2002; Costa et al., 2004; Fanti and Perez, 2004). Laboratory assays simulating water stress circumstances have aided to the identification of cultivars with an elevated level of resistance to such adverse conditions in cotton (Babu et al., 2014 and Megha et al., 2017) and other crops, such as maize (Tonin et al., 2000) and rice (Pirdashti et al.,_2003). However, the genotypic differences observed at seedling stage in hydroponic experiments may not necessarily correspond to those observed at the 
reproductive stage in the field (Zhu, 2001).

Throughout breeding for improving productivity under stress, two different points of view are considered: selection for high potential yield, accepting the hypothesis that if the yield of a genotype is increased in optimum conditions it will also be increased in non-optimum conditions, or selection for high yield under stress conditions (Blum, 1979).

In order to improve yield under drought conditions such new cultivars, two basic requirements must be available. Firstly, there must be sufficient variability for water stress tolerance in the crop as a whole, and secondly, this variation must be genetically controlled. To develop cotton varieties for drought tolerance, the first step in breeding program is to determine suitable parents. Thus, the main objectives of this work were to: A- determine the genotypic variability between cotton genotypes in response to water deficit stress B- detect the most suitable genotypes under water deficit condition for further using by cotton breeders $\mathrm{C}$ - evaluate seedling screening for water stress under hydroponic condition D- detect the most suitable selection criteria for water deficit and to test selection hypothesis (selection under optimum or water stress conditions).

\section{MATERIALS AND METHODS}

In the present study, two concurrent trials were conducted at Sakha Agric. Res. Station (seedling trail) at laboratory and (field trail) during 2016 and 2017 seasons.

\section{Seedling trial:}

In this trial, seeds of sixteen cotton varieties (Table 1) representing a high range of cotton characteristics, were germinated vertically in two layer filter paper sheets at $25^{\circ} \mathrm{C}, 10$ days after sowing the sheets were unrolled and the seeds that had produced normal seedlings were transferred to hydroponic culture of aerated box according to Babu et al., 2014, containing Hogland nutrient solution for control treatment $(0.0 \mathrm{MPa})$ and with polyethylene glycol (PEG-6000) solution with final osmotic potential -0.40 $\mathrm{MPa}$ for water deficit stress treatment, the concentration of PEG-6000 required to obtain this value of osmotic potential was determined by the equation of Michel and Kaufmann (1973).

After 18 days the following seedling traits were measured: root length $(\mathrm{cm})$ (RL), root fresh weight (g) (RFW), root dry weight (g) (RDW), shoot length (cm) (SHL), shoot fresh weight (g) (SHFW) and shoot dry weight (g) (SHDW). Five seedlings from each variety and replication were uprooted and washed with tap water. Clean and blotted dry seedlings were dissected at the collar point to separate shoot and root. Length of shoots and roots seedlings was measured and fresh weight was taken immediately. Shoots and roots were separately packed in a labeled paper bag, placed in an oven at $70^{\circ} \mathrm{C}$ for $48 \mathrm{~h}$ and dry weights of roots and shoots were taken, this trial was carried out during 2016 and 2017 seasons.

\section{Field trial:}

The same sixteen cotton varieties were evaluated under two water regimes well-watered (normal irrigation) and water-limited (deficit irrigation), in the field during 2016 (Y1) and 2017 (Y2) at Sakha Agric. Res. Station.

The two water regimes:

- Well-watered. One irrigation at planting and 6 subsequent irrigations as required for normal crop growth and development.

- Water-limited. One irrigation at planting and three supplemental irrigations 25 , 40 and 55 days after planting. 
M.A.A. El-Dahan, et al.,

Table 1. Pedigrees of the 16 cotton genotypes used in this study.

\begin{tabular}{|c|c|c|c|c|c|c|c|}
\hline No. & Genotype & Pedigree* & Category & No. & Genotype & Pedigree* $^{\star}$ & Category \\
\hline 1 & Giza 45 & $\begin{array}{c}\text { Giza } 28 x \\
\text { Giza } 7\end{array}$ & ELS & 9 & Giza 90 & $\begin{array}{l}\text { Giza } 83 x \\
\text { Dendera }\end{array}$ & LS \\
\hline 2 & Giza 70 & $\begin{array}{l}\text { Giza 59A x } \\
\text { Giza 51B }\end{array}$ & ELS & 10 & Giza 92 & $\begin{array}{c}\text { Giza } 84 \text { x (Giza 74 } \\
\text { x Giza 68) }\end{array}$ & ELS \\
\hline 3 & Giza 77 & $\begin{array}{c}\text { Giza } 70 x \\
\text { Giza } 68\end{array}$ & ELS & 11 & Giza 93 & (G. 77 x Pima S6 ) & ELS \\
\hline 4 & Giza 80 & $\begin{array}{c}\text { Giza } 66 x \\
\text { Giza } 73\end{array}$ & LS & 12 & Giza 94 & Giza 86 x 10229 & LS \\
\hline 5 & Giza 86 & $\begin{array}{c}\text { Giza } 75 x \\
\text { Giza } 81\end{array}$ & LS & 13 & $\begin{array}{c}\text { Giza } 89 x \\
\text { Giza } 86\end{array}$ & Giza 89 x Giza 86 & LS \\
\hline 6 & Giza 87 & $\begin{array}{l}\text { Giza } 77 \text { x } \\
\text { Giza 45-A }\end{array}$ & ELS & 14 & $\begin{array}{c}\text { Ashmouni } \\
\text { (Giza 19) }\end{array}$ & $\begin{array}{l}\text { Selected from } \\
\quad \text { Giza } 2\end{array}$ & LS \\
\hline 7 & Giza 88 & $\begin{array}{l}\text { Giza } 77 \text { x } \\
\text { Giza 45-B }\end{array}$ & ELS & 15 & $\begin{array}{l}\text { Menoufi } \\
\text { (Giza 36) }\end{array}$ & Wafeer x Sakha 3 & ELS \\
\hline 8 & Giza 89 & $\begin{array}{c}\text { Giza } 75 x \\
\text { Russian-6022 }\end{array}$ & LS & 16 & Suvin & $\begin{array}{c}\text { Indian variety } \\
\text { (Sujata x Vincent) }\end{array}$ & LS \\
\hline
\end{tabular}

* Pedigree information from Abdel-Salam (1999).

During both seasons each water regime experiment was conducted using a split-plot design with four replications were used with water regimes as the main plot and genotypes were randomly assigned as the sub-plots. Each plot consisted of one row of $\mathbf{5 . 0}$ meter long with $30 \mathrm{~cm}$ hill space, while row to row width was $70 \mathrm{~cm}$ apart. Two plants were left per hill at thinning time. The experiment received the recommended agronomic treatments of the commercial area.

Plants were picked by hand, the central ten guarded plants were used to determine the following yield and yield component traits: seed cotton yield (g)/plant (SCY/P), lint cotton yield (g)/plant (LCY/P), bolls/plant (B/P), boll weight (g) (BW), lint percentage (L\%), seed index (g) (SI) and lint index (g) (LI). Five of these central ten guarded plants were used to determine the following vegetative and morphological traits: plant height $(\mathrm{cm})(\mathrm{PH})$, number of vegetative branches/ plant (VB/P), number of fruiting branches/ plant $(F B / P)$, leaf area $\left(\mathrm{cm}^{2}\right)$ (LA), leaf fresh weight (g) (LFW) and leaf dry weight (g) (LDW). (Leaf traits were carried out on the fourth leaf from plant tip.

Fiber sample of each genotype and treatment were used to measure micronaire reading (MR), fiber length at $2.5 \%$ span length $(2.5 \% \mathrm{SL})$ in $\mathrm{mm}$, maturity ratio $(\mathrm{M})$ and pressley index $(\mathrm{PI})$ during the two seasons.

Drought susceptibility index (DSI) was calculated for yield modifying original Fischer \& Maurer, (1978) equation to detect genotype water stress susceptibility as:

$D S I=1-\left(Y_{D} / Y p\right) / S I$, while $S I=1-\left(\hat{Y}_{D} / \hat{Y} p\right)$

Whereas $S I$ is stress intensity and $\hat{Y}_{D}$ and $\hat{Y} p$ are the means of all genotypes under water stress and normal conditions, respectively. 
Statistical analysis:

The recorded data were subjected to analysis of variance technique (Steel \& Torrie, 1960) to obtain level of significance among the genotypes and water regime.

Correlation between all the studied traits under normal and water stress conditions was calculated; path coefficient analysis as formulated by Dewey and Lu (1959) was estimated for yield, yield components and some vegetative traits. Factor analysis of the contributed characters was expressed with eigen value and manifested in eigen vector for yield, yield components and some vegetative traits in each factor (Hair et al., 1987). All these computations were performed using SPSS (1995) computer procedure.

\section{RESULTS AND DISCUSSIONS Mean square and means}

\section{Seedling traits:}

Analysis of variance for seedling traits during growing seasons 2016 and 2017 revealed significant $(P \leq 0.05)$ variation with respect to water regimes, genotypes and the interaction of these two seasons (Table 2). However, the water regime was not significant for SHDW in 2016. Similar results were reported by Pettigrew, (2004a); Pettigrew, (2004b) and Başal et al. (2005).

Means of the relative reduction due to water stress (RD \%) in seedling traits over two years (Table 3) ranged from $15.2 \%$ for SHL to $21.7 \%$ for SHFW, similar finding were reported by Carlos et al., (2011) studying on exposing the cotton seedlings with different levels of PEG6000 revealed that differential viability and vigor between cultivars were observed under the water stress levels. Regarding to root traits, G.93 exhibited higher values under water stress for root traits. However, G. 80 was most affected genotype by water stress. In respect of shoot traits, G. 88, G. 93, and Suvin were less affected by water stress; in the otherwise G. 89 was most affected by water stress and showed reduction to $37.6 \%, 52.9 \%$ and $48.1 \%$ for SHL ,SHFW and SHDW, respectively. It could be concluded that the genotypes G.93 was less affected by water stress for seedling traits. Although the relative reduction (RD\%) of G. 94 was high for all seedling traits and considered as water deficit affected genotype, but its means performance maintained as one of the highest genotype under water deficit stress across all seedling traits, also Menoufi genotype showed the same trend for root traits.

\section{Vegetative traits}

Analysis of variance for vegetative traits during growing seasons 2016 and 2017 revealed significant $(P \quad \leq 0.05)$ variation with respect to water regimes except for LA during 2016; and LFW and LDW during 2017, genotypes except for LA during 2016 and the interaction of these two parameters except for $\mathrm{PH}$ during two years and LA during 2017 (Table. 2).

Mean values of vegetative traits of the genotypes in the well-watered and water limited regimes for 2016 and 2017 are presented in Table 3 . Means of the relative reduction due to water stress (RD $\%$ ) in vegetative traits over two years (Table 3) ranged from $12.31 \%$ for LFW to $36.80 \%$ for $\mathrm{FB} / \mathrm{P}$, similar results of the effect of water deficit on vegetative traits in cotton are revealed by (McMichael and Hesketh, 1982; Jordan, 1986; Turner et al., 1986; Ball et al., 1994; Gerik et al., 1996; Arbab et al., 2015 ). Regarding to $\mathrm{PH}$ and $\mathrm{FB} / \mathrm{P}$, the relative reduction (RD\%) revealed that G.89xG.86 genotype was the least affected by water stress, but G. 87 was the most affected one. Also, G.89, G.93 and G.77 were less affected by water stress for VB/P, but $G$. 80 and Menoufi were the most affected 
M.A.A. El-Dahan, et al.,

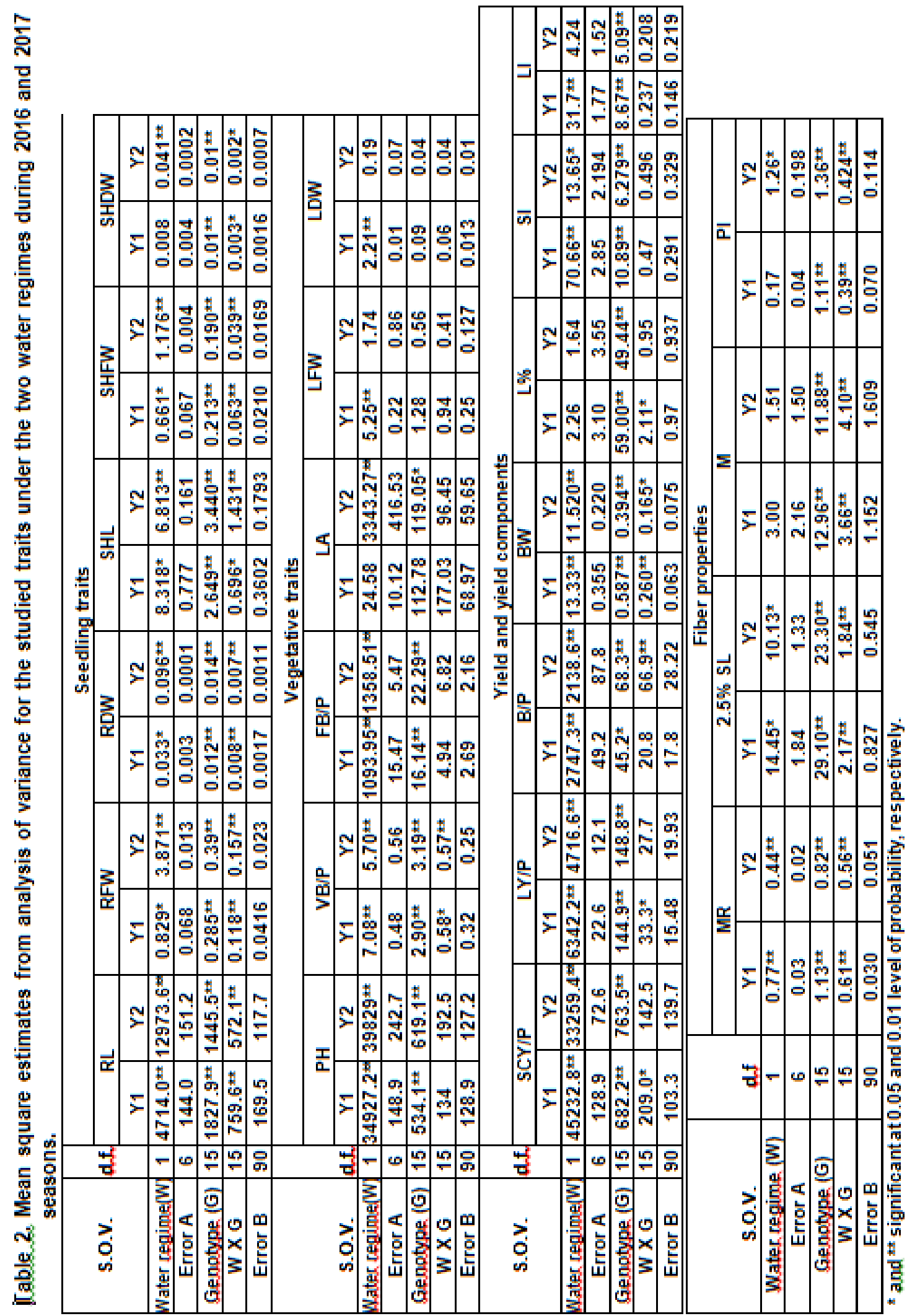




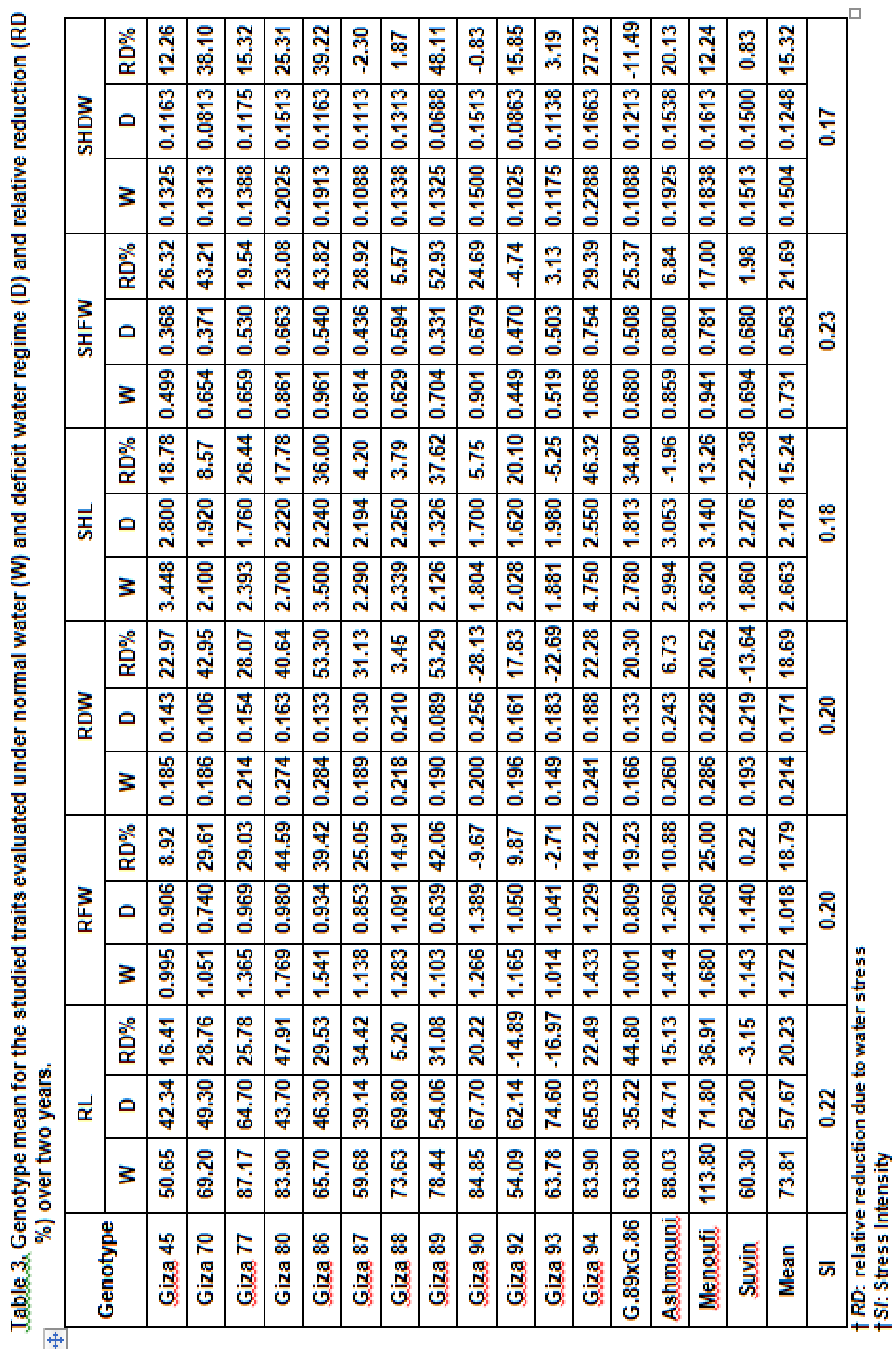




\begin{tabular}{|c|c|c|c|c|c|c|c|c|c|c|c|c|c|c|c|c|c|c|c|c|c|}
\hline \multirow{3}{*}{ בֵ } & ㅇํㅁ & $\stackrel{\mathscr{N}}{\sim}$ & $\stackrel{5}{\stackrel{2}{2}}$ & : & $\stackrel{\mathscr{O}}{\digamma}$ & $\begin{array}{l}q \\
\text { v }\end{array}$ & & ס & 잉 & $\stackrel{\dddot{C}}{=}$ & జ్ & 袽 & 올 & 롱 & 맘 & 守 & 穴 & i & \begin{tabular}{l}
$\infty$ \\
गु \\
\multirow{2}{*}{}
\end{tabular} & \multirow{3}{*}{ స્ّ } & \\
\hline & 口 & ษั่ & 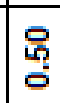 & 导 & 肯 & 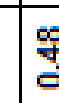 & & مִ & 苞 & 总 & 守 & 宛 & g. & 艿 & 号 & ్ㅠㅇ & $\mid \begin{array}{l}0 \\
0 \\
0\end{array}$ & 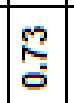 & 范 & & \\
\hline & 3 & 芯 & $\bar{\sigma}$ & 홍 & \% & $\underset{\infty}{\infty}$ & & $\overline{\mathscr{S}}$ & $\stackrel{\circ}{\circ}$ & 包 & ஜூ & 茼 & 웅 & 哭 & 营 & 宽 & $\mid \begin{array}{l}0 \\
0 \\
0 \\
0\end{array}$ & 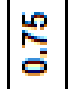 & 怘 & & \\
\hline \multirow{3}{*}{ ב3 } & ํํํ & \begin{tabular}{l} 
ণ \\
\multirow{\leftarrow}{*}{}
\end{tabular} & $\frac{\dot{\sigma}}{\sigma}$ & $\begin{array}{l}\text { षั. } \\
\text { 今 }\end{array}$ & $\begin{array}{l}\bar{\omega} \\
\dot{\omega}\end{array}$ & مొ & 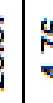 & $\stackrel{0}{\dot{q}}$ & 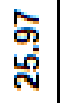 & 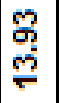 & ஸू & స్ & 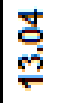 & $\underset{\sim}{\stackrel{5}{*}}$ & $\underset{\infty}{\mathscr{\infty}}$ & 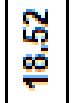 & $\begin{array}{l}\mathrm{N} \\
\mathrm{m} \\
\mathrm{q}\end{array}$ & 荤 & 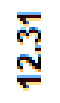 & \multirow{3}{*}{$\frac{m}{5}$} & \\
\hline & 0 & $\stackrel{\text { 吕 }}{\leftarrow}$ & 号 & 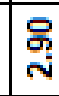 & $\stackrel{?}{?}$ & 옹 & & : & 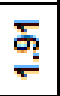 & స్త్ & 总 & $\stackrel{\infty}{\circ}$ & 오 & 苂 & స్ & กี & กี & 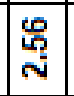 & $\stackrel{n}{i}$ & & \\
\hline & 3 & స్ & స్తి & ભ్ & 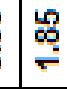 & $\stackrel{\infty}{\infty}$ & & 인 & 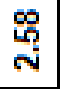 & 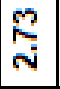 & స్తి & \%్ & స్ స్ & $\begin{array}{l}\text { 吕 } \\
\text { N }\end{array}$ & 导 & $\begin{array}{l}R \\
\text { in } \\
\text { in }\end{array}$ & స్ ָ̊ & 芯 & 品 & & \\
\hline \multirow{3}{*}{ 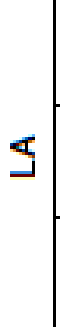 } & ㅇํㅁ & กั & ฟู & 吕 & $\stackrel{\varrho}{\circ}$ & 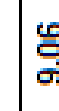 & & 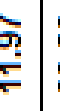 & స్లె & $\stackrel{g}{\stackrel{g}{*}}$ & $\underset{\sim}{\stackrel{\sigma}{\sigma}}$ & 莞 & 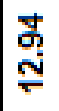 & $\begin{array}{l}\dot{O} \\
\dot{0}\end{array}$ & $\underset{\sim}{\mathbb{N}}$ & \begin{tabular}{l|}
$\mathscr{\mathscr { L }}$ \\
$\ddot{0}$ \\
$\infty$
\end{tabular} & $\left|\begin{array}{l}\mathscr{0} \\
0 \\
0 \\
0\end{array}\right|$ & 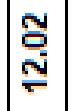 & 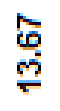 & \multirow{3}{*}{$\stackrel{⿱}{\circ}$} & \\
\hline & 0 & 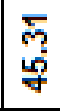 & 总 & 守 & \%ั & ร̃ & & 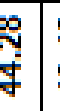 & $\begin{array}{l}\text { 栄 } \\
\text { ᄋ़ }\end{array}$ & $\begin{array}{l}\text { क्ष } \\
\text { ô }\end{array}$ & 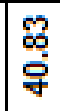 & $\mid \begin{array}{c}\mathscr{R} \\
\text { T. } \\
\text { OQ }\end{array}$ & 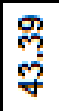 & 过 & 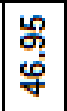 & 움 & $\mid \begin{array}{l}0 \\
\\
\dot{y}\end{array}$ & $\begin{array}{l}0 \\
0 \\
\dot{q}\end{array}$ & $\begin{array}{l}\text { 号 } \\
\text { 与. }\end{array}$ & & \\
\hline & 3 & 蟋 & 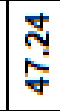 & 尽 & $\begin{array}{l}\text { જू } \\
\text { \& }\end{array}$ & 踏 & & בֶ. & $\begin{array}{l}\text { ¿ே. } \\
\stackrel{0}{0}\end{array}$ & 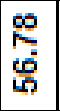 & సু & గ్రి & $\begin{array}{l}\text { 怘 } \\
\text { gं }\end{array}$ & $\begin{array}{l}\text { 召 } \\
\text { 点 }\end{array}$ & $\begin{array}{l}\stackrel{2}{a} \\
\text { go } \\
\text { gat }\end{array}$ & 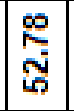 & $\begin{array}{l}\text { 员 } \\
\text { 呙 }\end{array}$ & 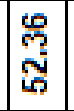 & 尔 & & \\
\hline \multirow{3}{*}{ 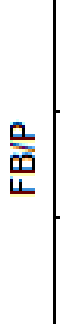 } & ㅇํㅁ & గ్రి & ?े & $\frac{d}{\dot{g}}$ & 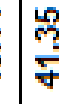 & รี & 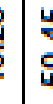 & : & $\begin{array}{l}\text { R } \\
\text { लें }\end{array}$ & 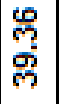 & ๙ั & 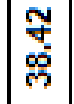 & 总 & $\begin{array}{l}R \\
\text { i } \\
\text { in }\end{array}$ & 导 & సু & $\begin{array}{l}\infty \\
\tilde{m} \\
\tilde{m} \\
\tilde{m}\end{array}$ & 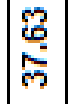 & $\begin{array}{l}\text { 吕 } \\
\text { 心 }\end{array}$ & \multirow{3}{*}{ 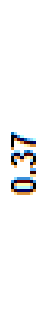 } & \\
\hline & 0 & 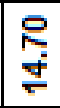 & 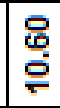 & \%ั & ले & 응 & & 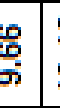 & $\stackrel{\oplus}{\circ}$ & 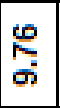 & స్్ㅇ & নু & $\stackrel{\oplus}{\sigma}$ & 융 & $\begin{array}{l}\mathscr{0} \\
\stackrel{\leftrightarrow}{\circ}\end{array}$ & 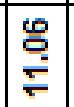 & 号 & 邑 & ్ㅜㅇ & & \\
\hline & 3 & 옹 & 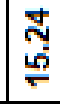 & $\begin{array}{l}\text { 苟 } \\
\stackrel{0}{\circ}\end{array}$ & $\stackrel{\mathscr{O}}{\stackrel{\mathscr{C}}{\digamma}}$ & $\begin{array}{l}0 \\
0 \\
0\end{array}$ & & 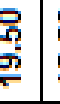 & 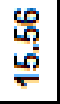 & 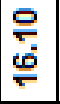 & 兽 & 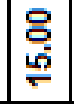 & 冚 & \begin{tabular}{l}
$\stackrel{0}{T}$ \\
\multirow{2}{*}{}
\end{tabular} & \begin{tabular}{l}
$\stackrel{\mathscr{O}}{+}$ \\
\multirow{+}{*}{}
\end{tabular} & $\begin{array}{l}\text { ్ㅗ } \\
\text { ळ }\end{array}$ & 苂 & 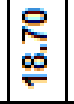 & $\begin{array}{l}R \\
\stackrel{0}{\circ}\end{array}$ & & \\
\hline \multirow{3}{*}{ 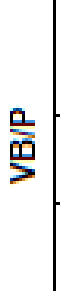 } & ํํㅁ & 志 & 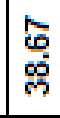 & $\begin{array}{l}\text { \% } \\
\text { o }\end{array}$ & 悉 & $\begin{array}{l}J \\
\text { J } \\
\text { f }\end{array}$ & & के & $\frac{\infty}{\grave{d}}$ & $\stackrel{\text { 움 }}{\frac{1}{7}}$ & ్ㅗㄹ & $\begin{array}{l} \\
\stackrel{\circ}{\circ} \\
\stackrel{\circ}{\circ}\end{array}$ & 응 & స్ & $\stackrel{?}{\stackrel{?}{*}}$ & $\begin{array}{l}\text { ஜू. } \\
\text { 今ू }\end{array}$ & 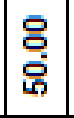 & స్ి & స్ & \multirow{3}{*}{ స్ } & \\
\hline & 0 & $\stackrel{\leftrightarrow}{\circ}$ & 楽 & 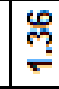 & 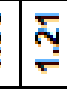 & $\stackrel{\infty}{2}$ & & סִ & $\stackrel{\circ}{\circ}$ & $\stackrel{9}{\check{-}}$ & $\stackrel{\text { श्? }}{\leftarrow}$ & 寽 & \%ั. & $\stackrel{\circ}{\circ}$ & ্ָ & 员 & 岕 & 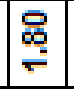 & ?़ & & \\
\hline & 3 & $\stackrel{\infty}{\circ}$ & స్ & $\stackrel{\circ}{8}$ & 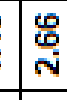 & 我 & & 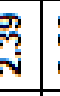 & 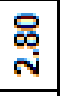 & 용 & 象 & $\stackrel{\mathscr{2}}{\mathscr{2}}$ & \%ัم & 웅 & \begin{tabular}{|l|}
$\mathscr{B}$ \\
$\stackrel{\text { N }}{ }$ \\
\end{tabular} & \begin{tabular}{|l|}
$\stackrel{8}{\circ}$ \\
0 \\
\end{tabular} & $\begin{array}{l}\text { 员 } \\
\end{array}$ & 旁 & $\stackrel{\circ}{\circ}$ & & \\
\hline \multirow{3}{*}{ II } & ㅇํㅁ & $\begin{array}{l}\text { o్ } \\
\text { : }\end{array}$ & ब̊ & ঙ্ল & నू & $\mathrm{m}$ & & $\stackrel{5}{\square}$ & $\overline{\dot{m}}$ & $\begin{array}{l}\mathscr{0} \\
\dot{0}\end{array}$ & 我 & 怘 & 옹 & ন & 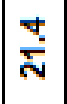 & 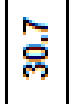 & జ̊ & 㶽 & న్ని & \multirow{3}{*}{ ম্ণু } & \\
\hline & 0 & ஷ̊ & జ̊ & "ి & జ్ & 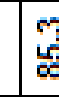 & & $\hat{\sigma}$ & $\begin{array}{l}0 \\
\stackrel{0}{0} \\
0\end{array}$ & 管 & 总 & 色 & 管 & 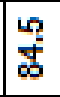 & ตุ: & $\frac{\text { ș }}{\sigma}$ & $\begin{array}{l}\infty \\
\tilde{~}\end{array}$ & 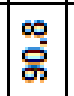 & ณุ & & \\
\hline & 3 & 웅 & $\stackrel{\infty}{\stackrel{\infty}{ }}$ & స్ & $\underset{\infty}{\mp}$ & 2 & רั & בُ & $\stackrel{\sim}{\stackrel{\Xi}{\circ}}$ & $\stackrel{\sim}{\stackrel{2}{*}}$ & $\stackrel{+}{\circ}$ & 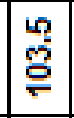 & $\begin{array}{l}\text { og } \\
\text { of } \\
\text { o }\end{array}$ & ํㅜㄹ & $\stackrel{\vec{m}}{\rightleftarrows}$ & 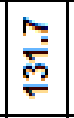 & $\stackrel{\sim}{\frac{\pi}{5}}$ & 옹 & 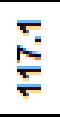 & & \\
\hline & 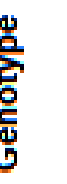 & 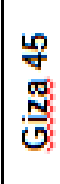 & $\begin{array}{l}\text { R } \\
\text { N } \\
\stackrel{N}{0}\end{array}$ & $\begin{array}{l}N \\
g \\
\mathcal{N}\end{array}$ & $\begin{array}{l}\text { \& } \\
\text { : } \\
\frac{N}{0}\end{array}$ & $\begin{array}{l}\mathscr{\infty} \\
\mathbb{0} \\
\mathbb{N} \\
: \mathcal{U}\end{array}$ & & 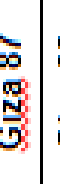 & 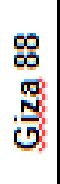 & 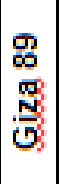 & 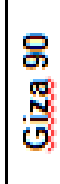 & $\begin{array}{l}\tilde{\alpha} \\
\alpha \\
\tilde{N} \\
\tilde{N}\end{array}$ & 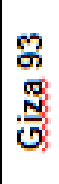 & 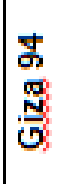 & 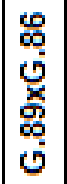 & 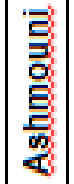 & 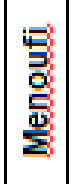 & ई & 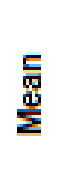 & $\bar{\kappa}$ & \\
\hline
\end{tabular}




\begin{tabular}{|c|c|c|c|c|c|c|c|c|c|c|c|c|c|c|c|c|c|c|c|c|}
\hline \multirow{3}{*}{$\Xi$} & 啇 & $\underset{\infty}{\infty}$ & 몽 & $\stackrel{\vec{m}}{\vec{m}}$ & $\stackrel{\infty}{\check{C}}$ & $\stackrel{\text { n? }}{2}$ & $\begin{array}{l}\stackrel{\infty}{\infty} \\
\stackrel{\infty}{\sigma}\end{array}$ & $\stackrel{?}{\stackrel{9}{\leftarrow}}$ & $\stackrel{\circ}{\circ}$ & ำ & $\stackrel{\text { g }}{\sim}$ & gi & $\stackrel{\infty}{\infty}$ & $\begin{array}{l}\mathscr{0} \\
\stackrel{6}{\circ}\end{array}$ & $\underset{\mathbf{I}}{\stackrel{乛}{\sim}}$ & 웅 & $\stackrel{\infty}{\text { N̊ }}$ & 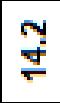 & \multirow{3}{*}{$\frac{d}{\sigma}$} & \\
\hline & 0 & 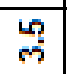 & in & 等 & $\stackrel{8}{8}$ & $\mathcal{F}$ & $\stackrel{\infty}{\sim}$ & I & f & $\frac{60}{8}$ & $\stackrel{\mathscr{\rho}}{\circ}$ & 品 & ลิ & \begin{tabular}{|l|}
$\infty$ \\
\multirow{q}{*}{}
\end{tabular} & लु & ? & $\frac{60}{8}$ & Y & & \\
\hline & 3 & m & f & 吕 & 5 & 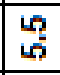 & d & \begin{tabular}{|l|}
0 \\
m
\end{tabular} & ปู & 范 & $\bar{q}$ & \begin{tabular}{|l|}
0 \\
\multirow{m}{*}{}
\end{tabular} & ?ִ & \begin{tabular}{|l|}
0 \\
0 \\
\end{tabular} & \begin{tabular}{|l|} 
\\
0 \\
\end{tabular} & $\begin{array}{l}0 \\
0 \\
\end{array}$ & $\begin{array}{l}0 \\
0 \\
\end{array}$ & $\frac{g}{8}$ & & \\
\hline \multirow{3}{*}{$\bar{\sigma}$} & ㅇํำ & $\stackrel{\sim}{\stackrel{2}{\leftarrow}}$ & مَ & \%̆ & $\stackrel{\circ}{\circ}$ & 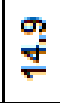 & $\underset{⿱ ㇒}{q}$ & $\hat{\infty}$ & $\stackrel{\text { वे }}{\Rightarrow}$ & $\underset{\mathrm{m}}{\stackrel{5}{\circ}}$ & స̃ & gr & 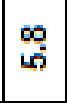 & \begin{tabular}{l|}
0 \\
웅
\end{tabular} & $\stackrel{+}{=}$ & \%̊ำ & 웅 & $\stackrel{\text { ğ }}{\stackrel{2}{2}}$ & \multirow{3}{*}{$\stackrel{m}{5}$} & \\
\hline & 0 & $\begin{array}{l}\infty \\
\dot{\omega} \\
\dot{\omega}\end{array}$ & $\begin{array}{l}\mathscr{0} \\
\dddot{6}\end{array}$ & 荧 & ?ִ & N & $\bar{\phi}$ & \begin{tabular}{|l|}
$\infty$ \\
0 \\
10
\end{tabular} & $\stackrel{⿱ 亠 乂}{i}$ & 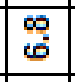 & 9 & $\overline{0}$ & $\stackrel{6}{\infty}$ & \begin{tabular}{|l|}
9 \\
\end{tabular} & \begin{tabular}{|c|} 
\\
$\infty$ \\
$\infty$
\end{tabular} & \begin{tabular}{|c|} 
\\
$\infty$ \\
$\infty$
\end{tabular} & \begin{tabular}{|l|l|}
$\infty$ \\
\end{tabular} & $\stackrel{N}{N}$ & & \\
\hline & 3 & $\begin{array}{l}\infty \\
\infty \\
\infty\end{array}$ & $\stackrel{\mathscr{O}}{\sim}$ & $\bar{\infty}$ & ?ִִֶ & $\stackrel{d}{0}$ & $\check{T}$ & 䓌 & $\stackrel{\mathscr{0}}{\mathscr{\infty}}$ & $\stackrel{\infty}{\sim}$ & $\stackrel{\leftrightarrow}{\sim}$ & 只 & $\bar{\sigma}$ & $\tilde{\sigma}$ & 응 & बे & $\stackrel{\circ}{\sigma}$ & $m_{\infty}^{2}$ & & \\
\hline \multirow{3}{*}{ ప̊ } & 商 & ले & 눙 & ભે & $\overline{\mathbf{i}}$ & $\stackrel{\text { ț }}{\circ}$ & $\overline{\mathrm{i}}$ & 宸 & ชู ซี & $\stackrel{\text { ?ִ }}{=}$ & $\stackrel{5}{-}$ & \begin{tabular}{|l|} 
\\
\\
0
\end{tabular} & $\stackrel{\infty}{\rightleftharpoons}$ & i & 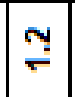 & $\underset{\gamma}{\stackrel{\Delta}{~}}$ & $\stackrel{n}{\circ}$ & T: & \multirow{3}{*}{ ¿ } & \\
\hline & 口 & ম્ల & 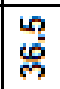 & 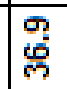 & 占 & స్ల & $\stackrel{m}{\bar{m}}$ & 总 & $\begin{array}{l}\infty \\
\infty \\
\infty\end{array}$ & 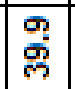 & 志 & लू & $\begin{array}{l} \\
\\
\end{array}$ & m & \begin{tabular}{l|}
0 \\
\\
$m$
\end{tabular} & m. & $\begin{array}{l} \\
\\
0\end{array}$ & 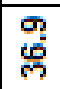 & & \\
\hline & 3 & m & $\begin{array}{l}m \\
0 \\
0 \\
\end{array}$ & 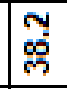 & $\begin{array}{l}\text { m? } \\
\text { ğ } \\
\end{array}$ & क्ष & 空 & $\overline{\dot{\phi}}$ & ஸ़े & 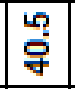 & $\begin{array}{l}\text { 웅 } \\
\text { ले }\end{array}$ & ్ㅗㄱ & \begin{tabular}{|c|}
$\stackrel{\infty}{q}$ \\
$\stackrel{9}{q}$
\end{tabular} & \begin{tabular}{|l|}
0 \\
0 \\
0 \\
\end{tabular} & लू & $\begin{array}{l} \\
\text { mo } \\
0 \\
m\end{array}$ & m & $\overline{\mathrm{m}}$ & & \\
\hline \multirow{3}{*}{ 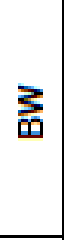 } & ڤั & 울 & مُ & $\stackrel{\mathscr{N}}{\stackrel{\leftrightarrow}{*}}$ & 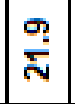 & ָุ & 品 & $\frac{1}{8}$ & $\stackrel{m}{\infty}$ & 웅 & 突 & 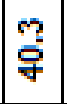 & 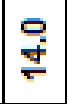 & 응 & $\overleftarrow{\sigma}$ & ปู & กี & $\stackrel{\square}{\sim}$ & & \\
\hline & 0 & $\overline{\mathrm{N}}$ & d & $\stackrel{\mathscr{N}}{ }$ & N & $\stackrel{\text { กุ }}{\mathrm{N}}$ & i̊ & 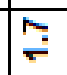 & No & ปู & $\stackrel{d}{d}$ & $\stackrel{\varphi}{\circ}$ & $\ddot{\sim}$ & $\tilde{N}$ & \begin{tabular}{|c|}
$\dot{\sim}$ \\
\end{tabular} & $\stackrel{\infty}{\sim}$ & 总 & กั & & \\
\hline & 3 & 웅 & $\tilde{N}$ & Oे & g & ก) & î & ) & Oे & g & ભั & $\stackrel{\omega}{i}$ & 웅 & $\bar{m}$ & テุ & mi & m & g̊ & & \\
\hline \multirow{3}{*}{ 号 } & ั̊ำ & $\stackrel{\text { 융 }}{\circ}$ & 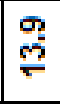 & 兽 & f & 寅 & 吕 & $\begin{array}{l}0 \\
\\
\dot{q}\end{array}$ & ణ్ల & 象 & ले & 告 & œ & \begin{tabular}{|l|} 
\\
$\infty$ \\
$\infty$
\end{tabular} & mon & 吕 & $\stackrel{\infty}{\text { N }}$ & $\frac{N}{m}$ & \multirow{3}{*}{ ָู } & \\
\hline & 0 & 염 & Бे & $\stackrel{+}{\stackrel{2}{*}}$ & 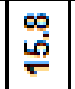 & $\check{\phi}$ & 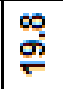 & $\check{\check{F}}$ & \begin{tabular}{|l}
$\mathscr{0}$ \\
$\stackrel{0}{\sigma}$
\end{tabular} & $\begin{array}{l}\stackrel{d}{*} \\
\stackrel{\infty}{\circ}\end{array}$ & $\begin{array}{l}\infty \\
\stackrel{6}{0} \\
\stackrel{0}{\sigma}\end{array}$ & 웅 & $\underset{\infty}{\mathscr{\infty}}$ & 守 & $\stackrel{\infty}{\stackrel{\infty}{*}}$ & $\stackrel{2}{5}$ & 号 & 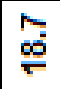 & & \\
\hline & 3 & 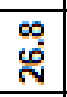 & $\overline{\tilde{z}}$ & \begin{tabular}{|l|}
$\infty$ \\
i̊ \\
\multirow{2}{*}{}
\end{tabular} & \begin{tabular}{|l|} 
n? \\
: \\
\end{tabular} & $\overline{\mathrm{N}}$ & \begin{tabular}{|l|}
5 \\
mp \\
\end{tabular} & 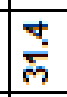 & 穴 & 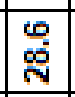 & స్ & స̊․ & 范 & $\frac{m}{\mathrm{~m}}$ & \begin{tabular}{|l|}
\multirow{2}{*}{} \\
: \\
\end{tabular} & 咅 & \begin{tabular}{|l|} 
i \\
i
\end{tabular} & 守 & & \\
\hline \multirow{3}{*}{ 은 } & 啇 & $\begin{array}{l}0 \\
0 \\
0\end{array}$ & m & 管 & 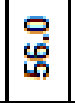 & $\bar{j}$ & 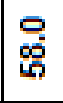 & 守 & గึ⿵冂 & 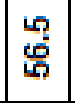 & 安 & 웅 & $\overline{0}$ & $\overline{\text { s. }}$ & 垈 & 岱 & $\stackrel{\mathscr{O}}{\text { f }}$ & $\stackrel{\infty}{5}$ & \multirow{3}{*}{ 菅 } & \\
\hline & 0 & m & 융 & $\overline{\mathfrak{I}}$ & $\stackrel{\square}{\rightleftarrows}$ & 뭉 & $\hat{\infty}$ & $\begin{array}{l}m \\
\infty \\
\infty\end{array}$ & 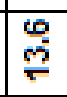 & ले & $\stackrel{\circ}{=}$ & $\stackrel{N}{\sim}$ & $\underset{F}{\stackrel{y}{*}}$ & \begin{tabular}{|l|}
\multicolumn{2}{|c|}{} \\
$\tilde{N}$
\end{tabular} & $\underset{+}{\stackrel{i}{*}}$ & $\underset{m}{m}$ & ูำ & 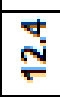 & & \\
\hline & 3 & $\begin{array}{l}\stackrel{0}{0} \\
\stackrel{0}{\sigma}\end{array}$ & $\stackrel{\text { ֻै }}{\infty}$ & 苍 & $\overline{\mathrm{N}}$ & $\bar{d}$ & กิ่ & ஸ̊ & กี & 吕 & 염 & $\begin{array}{l}+\infty \\
\infty\end{array}$ & 兽 & స్ల & 空 & $\stackrel{\infty}{\grave{N}}$ & 옴 & 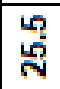 & & \\
\hline \multirow{4}{*}{ 号 } & फू & $\stackrel{\mathscr{C}}{\circ}$ & @ & ஜํㅜㅇ & $\stackrel{\circ}{\circ}$ & న్ & $\stackrel{m}{5}$ & స్ & 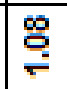 & 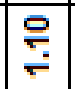 & $\stackrel{\circ}{\circ}$ & $\stackrel{\infty}{=}$ & $\stackrel{7}{\check{F}}$ & 㖞 & $\stackrel{5}{\circ}$ & 资 & \%ั. & $\stackrel{\circ}{\circ}$ & & \\
\hline & 商 & 势 & 守 & 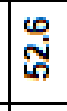 & 㝵 & 疋 & חั & 过 & 웅 & $\begin{array}{l} \\
0 \\
0\end{array}$ & 品 & m & $\frac{2}{6}$ & 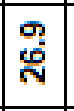 & \begin{tabular}{l|} 
\\
\multirow{4}{*}{}
\end{tabular} & 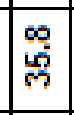 & 告 & $\frac{n}{5}$ & & ? \\
\hline & 0 & 㻤 & ?్ & ஹ્లె & $\overline{\text { s. }}$ & ले & $\stackrel{\sim}{\sim}$ & సู & 只 & $\overrightarrow{\text { हल }}$ & Б्ల. & i̊ & \begin{tabular}{|l|}
0 \\
足
\end{tabular} & \begin{tabular}{|c|}
\multirow{\delta}{*}{} \\
\end{tabular} & \begin{tabular}{|l|}
$\infty$ \\
\\
\end{tabular} & $\widehat{s}$ & $\frac{+}{\dot{y}}$ & 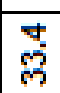 & & \\
\hline & 3 & $\begin{array}{l}0 \\
0 \\
0 \\
0\end{array}$ & Бे & $\overline{8}$ & ปู & $\check{\bar{\sigma}}$ & 它 & 它 & $\stackrel{9}{\kappa}$ & \begin{tabular}{l}
\multirow{2}{*}{} \\
ஸู
\end{tabular} & $\begin{array}{l}0 \\
0 \\
08 \\
0\end{array}$ & 害 & 吕 & 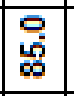 & \begin{tabular}{|l|}
0 \\
$\tilde{\infty}$ \\
\end{tabular} & m & 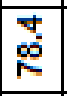 & $\begin{array}{l} \\
8 \\
80\end{array}$ & & \\
\hline \multicolumn{2}{|c|}{ 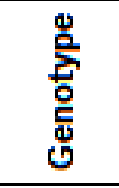 } & $\begin{array}{l}\text { 肙 } \\
\mathbb{g} \\
\frac{N}{\mathrm{G}}\end{array}$ & $\begin{array}{l}R \\
\text { R } \\
\stackrel{N}{0} \\
0\end{array}$ & $\begin{array}{l}\mathcal{E} \\
\mathbb{B} \\
\mathbb{N}\end{array}$ & 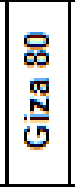 & 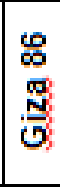 & $\begin{array}{l}\infty \\
\mathbb{8} \\
\mathbb{N} \\
\tilde{N}\end{array}$ & 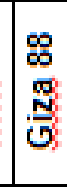 & 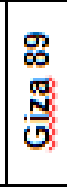 & 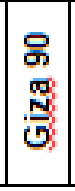 & 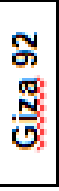 & 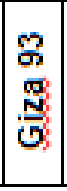 & 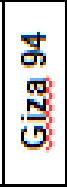 & 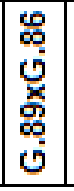 & 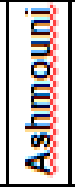 & 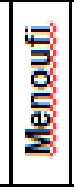 & 昰 & 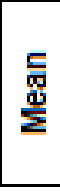 & $\bar{\omega}$ & 要点 \\
\hline
\end{tabular}


M.A.A. El-Dahan, et al.,

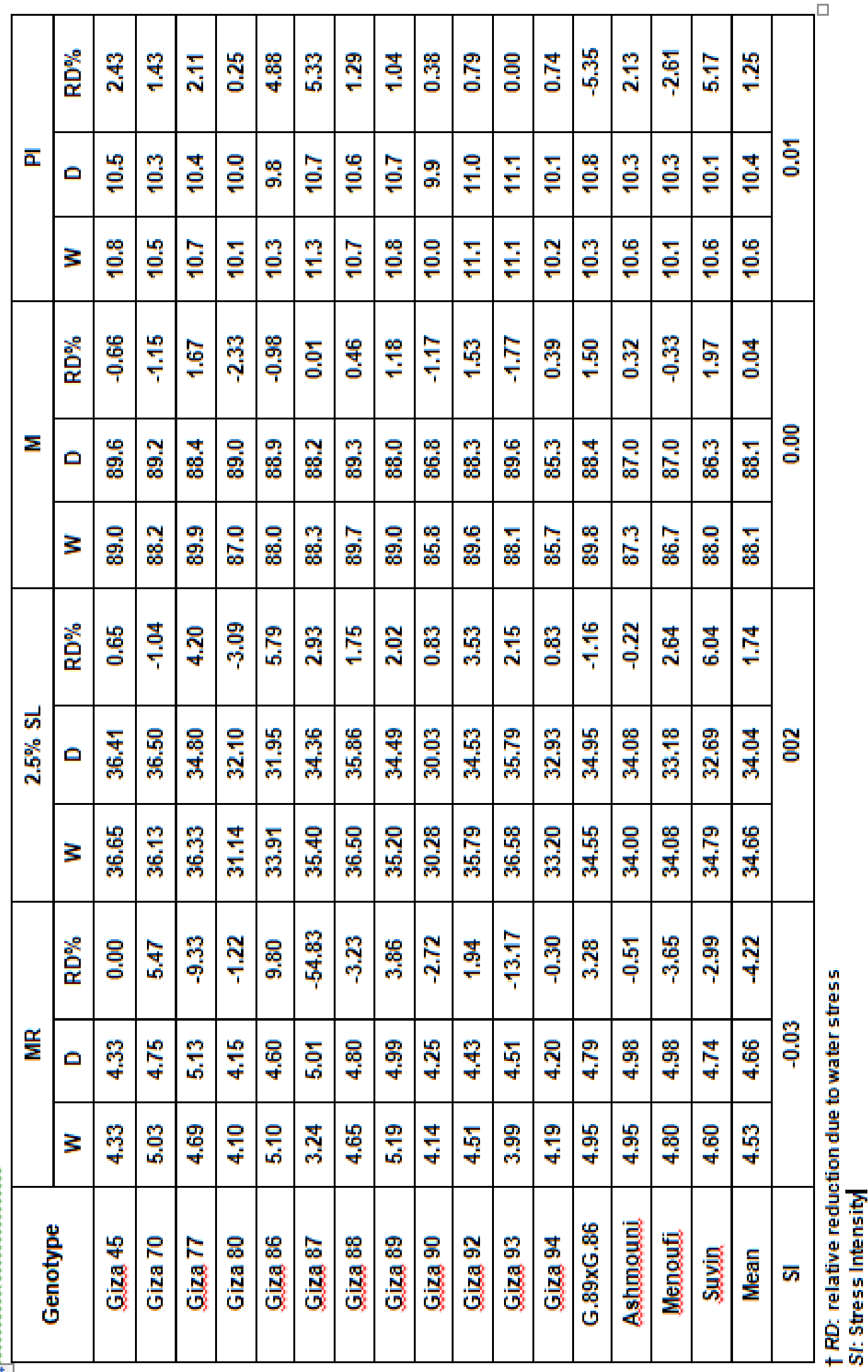


genotypes. For leaf traits, G.77and G.94 showed the highest means for LA and LFW under water deficit condition, where G.88, G.89, Menoufi and Suvin showed the highest means for LDW under water deficit condition. The results of vegetative traits concludes that G.77, G.94, G.89xG.86, Ashmouni, Menoufi and Suvin showed higher values under water deficit condition for most of vegetative traits.

\section{Yield and yield components}

The analysis of variance for yield and yield components traits during the growing seasons 2016 and 2017 are presented in Table 2. Water regime and genotype mean squares were significant $(P \leq 0.05)$ for all yield and yield components traits except for $L \%\left(Y_{1}\right.$ and $\left.Y_{2}\right)$ and $L I\left(Y_{2}\right)$ in water regime . In the same trend, the interaction of water regime $x$ genotype was significant for $S C Y / P, L Y / P$ and $L \%$ in $Y_{1}, B / P$ in $Y_{2}$ and $B W$ in both $Y_{1}$ and $Y_{2}$. Detecting of genotypic variability of yield and yield components were reported in many studies (Quisenberry et al., 1981; Pettigrew and Meredith, 1994; Lacape et al., 1998).

Mean values of yield and yield components in well watered and water limited regimes over two years are presented in Table 3 . The results revealed that all yield and yield components traits showed significant reduction under water limited regime except for L\%; relative reduction ranged from $51.6 \%$ for LY/P to $12.9 \%$ for SI. All genotypes revealed relative reduction under water limited regimes for all traits except for $L \%$. These results confirm the negative effect of water deficit on yield and yield components and the presence of genotypic variation for water stress tolerance in the examined materials, similar results of the effect of water deficit on yield and yield components traits in cotton are revealed by (Pettigrew, 2004b; Wang et al., 2004; Mert, 2005; Basal et al., 2009). Variation in SCY/P occurred among the 16 genotypes under a well-watered regime with values ranging from $50.1 \mathrm{~g} / \mathrm{P}$ for Giza 70 to $85.0 \mathrm{~g} / \mathrm{P}$ for G.89xG.86. When the genotypes experienced water-deficit stress, the genotype G.89xG.86 showed the highest yield and experienced the lowest reduction in yield with RD\% of $26.9 \%$, followed by Menoufi with RD\% of $35.8 \%$.

Biplot between SCY/P under water deficit stress and the control (no stress) is presented in figure 1. A significant positive relationship between yield under optimum condition and under water deficit stress was observed $(r=0.68$, $P<0.01, n=16$ ), supported the hypothesis that genotypic advantages selected under near-optimum growing conditions may be obtained under less favorable growing environments (Quisenberry et al., 1980). Genotypes G.89xG.86, Ashmouni, Suvin, G. 94 and G. 89 had the highest yield in both treatments. Genotypes G. 87, G. 88 and G. 93 suffered substantial yield losses under water-deficit than the other genotypes. Meanwhile, genotypes G.89xG.86, Menoufi, G. 86 and G. 70 were less affected by water stress.

The drought susceptibility index (DSI) was also calculated (Table 3 ) to provide an additional measurement of drought tolerance of the genotypes with respect to yield. DSI ranged from 0.53 to 1.26 , the relationship between DSI and the production under stressed conditions is presented in Figure 2. Drought stress tolerant genotypes were those with DSI values lower than the unit, while susceptible ones were those with DSI values greater than the unit. The result of 
a biplot analysis is shown in Figure 2 which is divided into four quadrants. In biplot quadrant I demonstrate four genotypes which are not only water stress tolerant but also give high seed cotton yield ((G.89xG.86), Suvin, Menoufi and G.86). Quadrant II, includes one genotype (G.70) which is fairly tolerant to water stress but produced lower production. Quadrant III, represents three genotypes which are susceptible to water stress but produced relatively high yield in limited water regime (G.89, G.94 and Ashmouni). Quadrant IV corresponds to susceptible eight genotypes with lower yields (G.45, G.77, G.80, G.87, G.88, G.90, G.92 and G.93). These results confirm that most of high productivity genotypes under water deficit condition belong to long stable cotton; and in the otherwise the most susceptible and low productivity genotypes belong to extralong staple cotton.

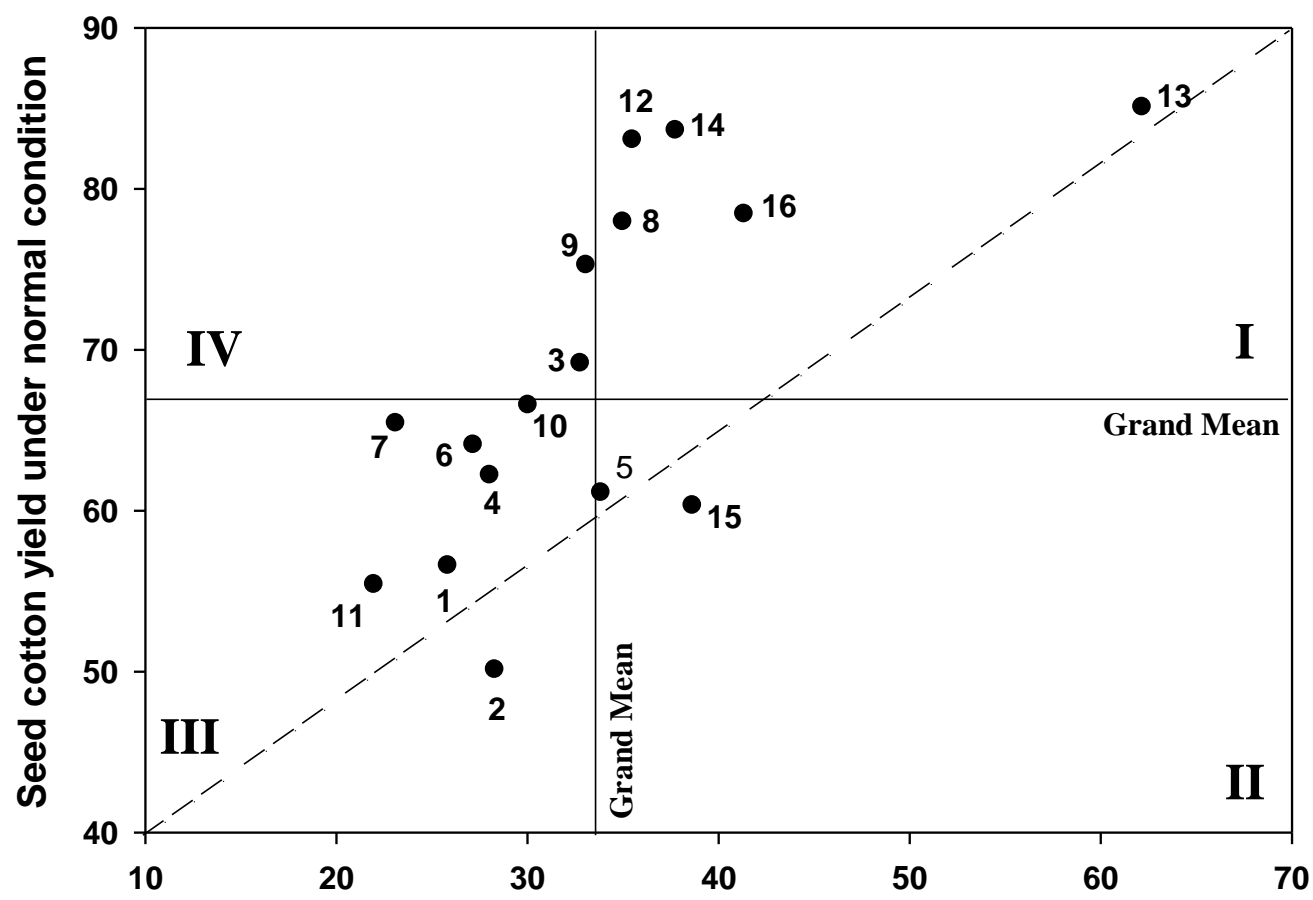

Seed cotton yield under water stress condition

1. Giza 45

2. Giza 70

3. Giza 77

4. Giza 80

5. Giza 86

6. Giza 87

7. Giza 88

8. Giza 89
9. Giza 90

10. Giza 92

11. Giza 93

12. Giza 94

13. Giza G89 x G86

14. Ashmouni

15. Menoufi

16. Suvin

Figure 1. Genotypic productivity of seed cotton under water stress conditions versus productivity under normal condition 
Biplot between seed cotton yield recorded under water defict conditions and the drought susceptibility index

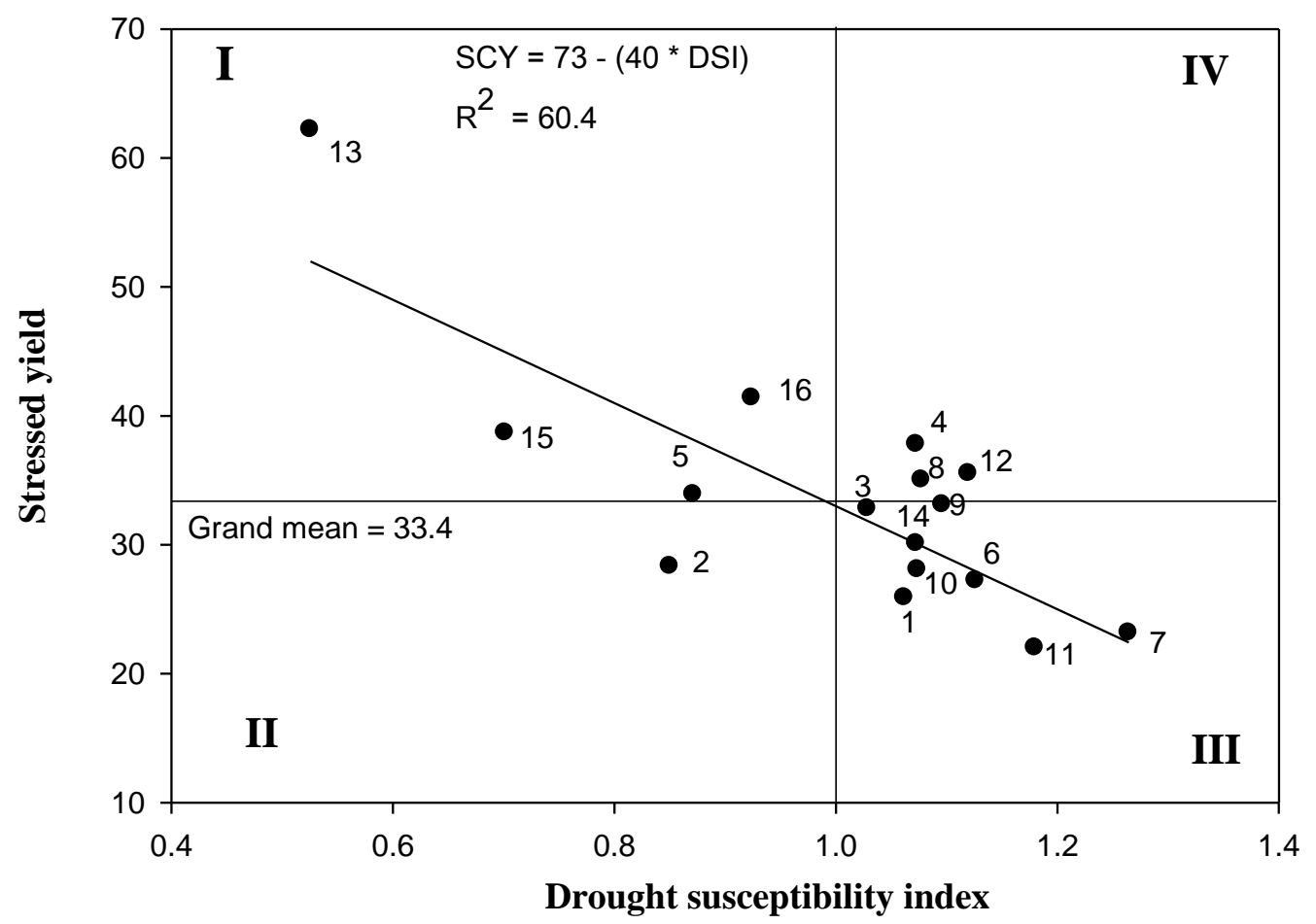
1. Giza 45
2. Giza 70
3. Giza 77
4. Giza 80
5. Giza 86
6. Giza 87
7. Giza 88
8. Giza 89

9. Giza 90
10. Giza 92
11. Giza 93
12. Giza 94
13. Giza G89 x G86
14. Ashmouni
15. Menoufi
16. Suvin

Figure 2. Biplot between seed cotton yield recorded under water deficit conditions and the drought susceptibility index

Non significant correlation between DSI and SCY/P under normal condition was detected (Table 4); however DSI showed significant negative correlation with SCY/P. $\mathrm{LY} / \mathrm{P}, \mathrm{B} / \mathrm{P}, \mathrm{BW}$ and $\mathrm{PH}$ under water stressed conditions ranged from $(r=-0.59, P<0.05$, for $B / P)$ to $(r=-0.79, P<0.001$, for SCY) (Table 4) also regression analysis between $D S I$ and seed cotton yield under water deficit stress was negative (Figure 2) and the determination coefficient was $60.4 \%$ confirming the negative relation between DSI and stressed yield. These results clearly suggested DSI as a useful predictor of drought tolerance in cotton and confirming the need of performing genotype evaluation under water stress when breeding for water deficit tolerance. These findings also supported by Rashid et al., (1999), Moinuddin et al., (2005), Ullah et al., (2006) and Sezener et al., (2015) they reported that DSI might provide a more effective mean to assess drought tolerance in crops. Regarding to G.89, G.94 and Ashmouni, these genotypes showed high relative reduction (RD\%) and values of DSI higher than the unit, and from these point of view are 
classified as susceptible genotypes, in the same time these genotypes had values of SCY/P exceeded the general mean of SCY/P under water deficit stress. These results suggest the possibility to use these genotypes as high yield potential genotypes under water deficit stress.

LY/P had approximately the same trend of SCY/P, and other yield components were also affected by water deficit stress, $L \%$ was the lowest affected trait, while some genotypes exhibited higher L\% under water stress than normal condition. G. 89, G. 92 and Menoufi showed higher $L \%$ under water limited stress than normal condition, where G. 45 and G. 77 were the most affected genotypes.

Regarding to B/P, G.45 and G. 70 showed the lowest reduction in bolls / plant under water deficit stress, but G. 80 and G. 88 showed the highest reduction under water stress.

G. 70 and G.89xG.86 showed the lowest reduction in BW under water deficit stress; but G. 88 and G.93 showed the highest reduction in BW.

Referring to SI and LI, G. 94 showed the highest value and low reduction under limited water regime; however Suvin showed the highest reduction.

These results for yield and its components clearly indicate a significant magnitude of variation in the response of various cotton genotypes to water stress, and in most cases G.89xG.86, Menoufi, Suvin and G. 86 showed the highest water deficit tolerance with acceptable production under limited water regime. On the other hand most of extra-long staple cotton varieties G.87, G.88 and G.93 were most susceptible to water deficit stress in production term.

\section{Fiber traits}

Analysis of variance for fiber traits under the growing seasons 2016 and 2017 revealed significant $(P \leq 0.05)$ variation with respect to water regimes except for maturity during two years and for $\mathrm{PI}$ in 2016. Both genotype and water regime $\mathrm{x}$ genotype mean squares were significant for all fiber traits under $Y_{1}$ and $Y_{2}$. (Table 2).

Mean values of fiber traits in well watered and water limited regimes over two years are presented in Table 3. Most of fiber traits exhibited relative reduction (RD\%), ranged from 0.04 for $M$ to -4.2 for MR, confirming the negative effect of water deficit on fiber properties. Similar results were obtained by Pettigrew, (2004b); Mert, (2005); Mahmood et al., (2006) and Osborne and Banks, (2006) . For Micronaire reading (lower values are desirable), G.77, G.87 and G.93 were the most affected genotypes by water deficit stress and showed higher values under water deficit stress, however G.70, G.86 and G.89 were less affected by water stress and showed lower values under water deficit stress.

Regarding to fiber length $(2.5 \% \mathrm{SL}), \mathrm{G} .77, \mathrm{G} .86, \mathrm{G} .92$ and Suvin were the most affected genotype, however G.70, G.80 and G.89xG.86 showed relatively higher $2.5 \% \mathrm{SL}$ under water deficit stress.

Most of genotypes exhibited low maturity change under water stress, indicating the little effect of water stress on maturity trait.

G.86, G.87 and Suvin were the most affected genotypes by water deficit stress exhibiting higher change for PI; however G.89xG.86 and Menoufi showed higher value for PI under water deficit stress. 
These results of fiber traits clearly indicate the effect of water deficit stress on these traits, and some of these traits were more affected than others. G.86 suffered reduction in $2.5 \% \mathrm{SL}$ and PI; G.87 suffered reduction in all fiber traits, however G.89xG.86 exhibited better results under water stress, in general most of the extra-long genotypes were more susceptible to water deficit stress than long stable genotypes.

\section{Correlation and Path analysis:}

Correlation coefficients between all the studied traits and DSI under well watered (upper value) and limited water regimes (lower values) over two years are presented in Table 4. Seedling traits showed positive correlations between most of their traits under the two regimes, all of the seedling traits tended to correlate negatively with VB/P under limited water regime showing significant for $R L$ under the two regimes and for SHFW under water limited regimes. Most of seedling traits tended to correlate positively with $L Y / P, L \%, S I$ and $\mathrm{LI}$ under well watered regime, however shoot traits showed this correlation trend with SI and LI under limited water regime. Most of fiber traits except MR, showed negative correlation with seedling traits specially root and shoot weights under both water regimes, indicating the possibility to use seedling traits to select for fiber traits under well water and limited water regimes.

In relation to vegetative traits, $\mathrm{PH}$ showed positive significant correlation with FB/P under the two water regimes and leaf traits also showed positive significant correlation with their traits. Also, PH showed positive significant correlation with yield and most of yield components under limited water regime; however LDW showed positive and significant correlation with BW, SI and $\mathrm{LI}$ under limited water regime. These results indicate the possibility to use PH and LDW as selection criteria to improve yield and some of yield components under limited water regime. Fiber traits did not exhibit significant correlation with any of vegetative traits.

Yield and yield components traits exhibited positive and significant correlation between their traits. Most of fiber traits except MR tended to correlate negatively with yield and yield components under the two water regimes, this negative correlation confirm the difficulty to improve productivity and fiber properties in the same time under water deficit stress. This finding concludes that, breeding method to improve productivity under water deficit stress should break the negative linkage between yield and fiber properties or at least maintain fiber properties out of deterioration.

The correlation coefficients between SCY/P under deficit water stress condition and yield components traits and some vegetative traits were partitioned into direct and indirect effects. The path coefficient analysis (Table 5 ) revealed positive and negative direct effect of traits on SCY/P. The highest direct effect on seed cotton yield was exhibited by B/P (1.362) followed by BW (0.908) and L\% (0.534). The highest indirect effect of most of yield and vegetative traits were through B/P and BW, however most of the studied traits exhibited negative indirect effect throw LY/P and LI. These results confirm that, selection to improve productivity under water deficit stress could be more effective through direct selection for boll number and boll weight, these two traits which consider the most important yield components under water deficit stress. Similar results were obtained by El-Dahan et al., (2002); Iqbal et al., (2006) and Ahuja et al., (2006).

\section{Factor Analysis:}

In order to identify vital components that contribute to total variation, factor analysis was conducted. Table 6 shows 
M.A.A. El-Dahan, et al.,

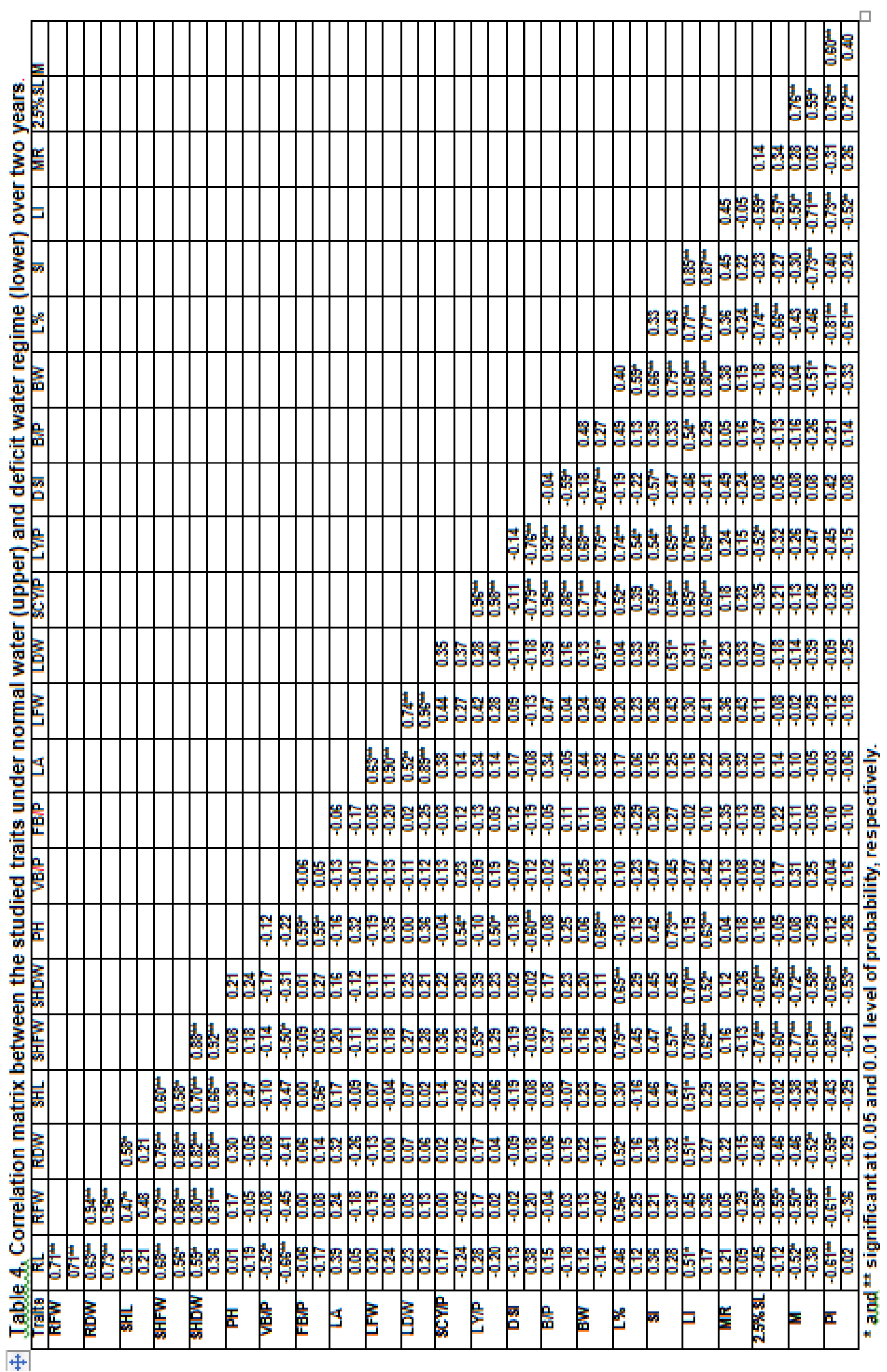


Table 5. Direct (diagonal) and indirect effects for yield and yield related traits on seed cotton yield under water deficit condition.

\begin{tabular}{|c|c|c|c|c|c|c|c|c|c|c|}
\hline Trait & LY/P & B/P & BW & L\% & SI & LI & PH & VB/P & FB/P & r (SCY) \\
\hline LY/P & -0.896 & -0.735 & -0.672 & -0.484 & -0.582 & -0.618 & -0.448 & -0.170 & -0.045 & $0.98^{\star *}$ \\
\hline B/P & 1.117 & 1.362 & 0.368 & 0.177 & 0.449 & 0.395 & 0.340 & 0.558 & 0.150 & $0.86^{\star *}$ \\
\hline BW & 0.681 & 0.245 & 0.908 & 0.536 & 0.718 & 0.727 & 0.618 & -0.118 & 0.073 & $0.72^{\star *}$ \\
\hline L\% & 0.288 & 0.069 & 0.315 & 0.534 & 0.230 & 0.411 & 0.069 & -0.123 & -0.155 & 0.39 \\
\hline SI & 0.166 & 0.084 & 0.201 & 0.110 & 0.255 & 0.222 & 0.186 & -0.115 & 0.069 & $0.64^{\star *}$ \\
\hline LI & -0.473 & -0.199 & -0.548 & -0.527 & -0.596 & -0.685 & -0.432 & 0.288 & -0.068 & $0.60^{\star *}$ \\
\hline PH & 0.106 & 0.053 & 0.144 & 0.028 & 0.155 & 0.134 & 0.212 & -0.047 & 0.125 & $0.54^{\star}$ \\
\hline VB/P & -0.008 & -0.017 & 0.006 & 0.010 & 0.019 & 0.018 & 0.009 & -0.042 & -0.002 & 0.23 \\
\hline FB/P & -0.001 & -0.003 & -0.002 & 0.008 & -0.007 & -0.003 & -0.015 & -0.001 & -0.026 & 0.12 \\
\hline
\end{tabular}

Table 6. Eigen values, percent variation and cumulative $\%$ for $\mathbf{1 0}$ factors.

\begin{tabular}{|c|c|c|c|}
\hline Factor & Eigen Value & Variation\% & Cumulative\% \\
\hline 1 & 5.26 & 52.61 & 52.61 \\
\hline 2 & 2.00 & 20.03 & 72.65 \\
\hline 3 & 1.59 & 15.87 & 88.51 \\
\hline 4 & 0.53 & 5.31 & 93.83 \\
\hline 5 & 0.39 & 3.86 & 97.68 \\
\hline 6 & 0.14 & 1.44 & 99.12 \\
\hline 7 & 0.08 & 0.76 & 99.88 \\
\hline 8 & 0.0107 & 0.1071 & 99.99 \\
\hline 9 & 0.0011 & 0.0111 & 100.00 \\
\hline 10 & 0.0001 & 0.0009 & 100.00 \\
\hline
\end{tabular}

total variance of each factor in percentage, which shows its importance in interpretation of total variation of data. Therefore, the contribution of each trait according to other traits is obtained. Three classes of independent factors were chosen based on Eigen value $>1$, which together compose $88.51 \%$ of total variation. Contribution of these three factors in total was 52.61, 20.03 and $15.87 \%$, respectively.

A principal factor matrix after Varimax rotation (Kaiser, 1958) for these three factors given in Table 7. To interpret the results, only those factors loading having greater values are considered. Factor 1 , which account for about $52.61 \%$ of the variation consists of LI, SI, L\%, BW, LY/P, SCY/P and PH. The suggested name of this factor is yield components due to the strong association between most of yield components and this factor. However the second factor which accounts for about $20.03 \%$ of the total variation was strongly associated with $B / P, S C Y / P$ and $L Y / P$ so the suggested name of this factor is yield factor. The third factor which accounts for about $15.87 \%$ of the total variance was strongly associated with $\mathrm{PH}$ and $F B / P$ and the suggested name of this factor is vegetative factor. Factor analysis exhibited the contribution of yield components in Factor 1 and 2 to improve productivity under deficit water stress. 
M.A.A. El-Dahan, et al.,

Table 7. Rotated factor loadings and communalities for yield and yield related traits.

\begin{tabular}{|c|c|c|c|c|}
\hline Trait & Communality & Factor 1 & Factor 2 & Factor 3 \\
\hline SCY/P & 0.981 & 0.535 & 0.823 & 0.135 \\
\hline LY/P & 0.995 & 0.621 & 0.780 & 0.026 \\
\hline B/P & 0.861 & 0.154 & 0.911 & 0.085 \\
\hline BW & 0.810 & 0.837 & 0.304 & 0.131 \\
\hline L\% & 0.815 & 0.766 & 0.106 & -0.466 \\
\hline SI & 0.897 & 0.870 & 0.133 & 0.350 \\
\hline LI & 0.947 & 0.963 & 0.124 & 0.074 \\
\hline PH & 0.895 & 0.595 & 0.154 & 0.719 \\
\hline VB/P & 0.802 & -0.549 & 0.706 & -0.052 \\
\hline FB/P & 0.848 & -0.019 & 0.047 & 0.920 \\
\hline
\end{tabular}

\section{REFERENCES}

Abdel-Salam, M.E. (1999).THE EGYPTIAN COTTON: Production, Quality and Marketing. Elkalema Press, 4, Ahmed Barada St. Giza-Cairo, Egypt.

Ahuja, S.L., L.S. Dhayal and P. Ram (2006). A correlation and path coefficient analysis of components in G hirsutum L. Hybrids by usual and fiber quality grouping. Turk. J. Agric. For., 30: 317-324

Arbab, S., Z. A. Baloch, A. Mahar, S. Otho, S. Kalhoro, A. Ali, F. Kalhoro, R. Soomro, F. Ali (2015). Effect of Water Stress on the Growth and Yield of Cotton Crop (Gossypium hirsutum L.). American Journal of Plant Sciences, 6, 1027-1039.

Babu, A. G., B. C. Patil and K. N. Pawar (2014). Evaluation of cotton genotypes for drought tolerance using PEG-6000 water stress by slanting glass plate technique. The Bioscan, 9 (2): 1419-1424.

Ball, R.A., D.M. Oosterhuis and A. Maromoustakos (1994). Growth dynamics of the cotton plant during water-deficit stress. Agron. J. 86:788795.

Başal, H., C.W. Smith, P.S. Thaxton and J.K. Hemphill (2005). Seedling drought tolerance in upland cotton. Crop Sci., 45: 766-771.

Basal, H., N. Dagdelen, A. Unay and E. Yilmaz (2009). Effects of deficit drip irrigation ratios on cotton (Gossypium hirsutum L.) yield and fiber quality. J. Agron. Crop Sci., 195: 19-29.

Blum, A. (1979). Genetic improvement of drought resistance in crops plants: a case for sorghum. In: Stress Physiology in Crops Plants (H. Mussell and R.C. Staples, eds.) pp. 430-445. Wiley- Interscience, New York.

Carlos, H. S., L. A. Riselane, P. D. Fernandes, E. P. Walter, H.G. Leonardo, M.A. Marleide and S.V. Murcia (2011). Germination of cotton cultivar seeds under water stress induced by polyethyleneglycol-6000. Sci. Agric. (Piracicaba, Braz.). 68(2): 131-138.

Costa, P.R., C.C. Custódio, N.B. Machado Neto and O.M. Marubayashi (2004). Water deficit induced by mannitol on soybean seed classified in different sizes. Revista Brasileira de Sementes 26:105-113. (in Portuguese with abstract in English).

Dewey, D.R. and K.H. Lu (1959). A correlation and path coefficient analysis of components of crested wheat grass seed production. Agron. J. 51(9): 515-518.

El-Dahan, M.A.A., M. López, E. O. Leidi and J.C Gutiérrez (2002). Genetic studies on drought tolerance in Upland Cotton (Gossypium hirsutum L.). Beltwide cotton conference, Atlanta G A- January 8-12. 
Fanti, S. C. and S. C. J. G. A. Perez (2004). Water stress and accelerated aging effects on the viability of osmoconditioned Chorisia speciosa seeds Pesquisa Agropecuária Brasileira, 38: 537-543.

Fischer, R.A. and R. Maurer (1978). Drought resistance in spring wheat cultivars. I., Grain yield response. Aust. J .Agric. Res. 29, 897-907.

Gerik, T.J., K.L. Faver, P.M. Thaxton and K.M. El-Zik (1996). Late season water stress in cotton: I. Plant growth, water use and yield. Crop Sci., 36:914-921.

Guinn, G. and J.R. Mauney (1984). Fruiting of cotton. Effects of plant moisture status and active boll load on boll retention. Agron. J. 76:94-98.

Hair, J.F., Jr. R.E. Anderson and R.L. Tatham (1987). Multivariate Data Analysis with Reading. MacMillan Publ., Co., New York.

Hsiao, T.C., E. Acevedo, E. Fereres and D.W. Henderson (1976). Stress metabolism: Water stress, growth and osmotic adjustment. Phil. Trans. R. Soc. Lond. B. 273:479-500.

Iqbal, M., K. Hayat, R. S. Khan, A. Sadiq and N. Islam (2006). Correlation and path coefficient analysis for earliness and yield traits in cotton (G. hirsutum L.). Asian J. Plant Sci.,5: 341-344

Jordan, W.R. (1986). Water deficits and reproduction. pp.63-73. In: J.R. Mauney and J.M. Stewart (ed.). Cotton Physiology. The Cotton Foundation, Memphis, Tenn.

Kaiser, H. F. (1958). The varimax criterion for analytic rotation in factor analysis. Psychometrika 23, 187-200.

Kramer, P.J. (1983). Water deficits and plant growth. pp: 342-389. In: P.J. Kramer (ed.). Water relations of plants. Academic Press, New York.

Lacape, M.J., J. Wery and D.J.M. Annerosa (1998). Relationship between plant and soil water status in five field-growing cotton (Gossypium hirsutum L.) cultivars. Field Crops Res., 57:29-48.

Larcher, W. (2000). Plant Ecophysiology. RIMA, São Carlos, SP, Brazil. 5 (31): 57-70.
Le Houerou, H.N. (1996). Climate changes, drought and desertification. J. Arid. Environ. 34:133-185.

Loka, D.A., D.M. Oosterhuis and G.L. Ritchie (2011). Water-Deficit Stress in Cotton. pp. 37-72. In: D.M. Oosterhuis (ed.). Stress Physiology in Cotton. The Cotton Foundation, Memphis, Tenn

Mahmood, S., M. Irfan, F. Raheel and A. Hussain (2006). Characterization of cotton (Gossypium hirsutum L.) varieties for growth and productivity traits under water deficit conditions. Int. J. Agric. Biol., 8: 796-800.

Massacci, A., S.M. Nabiev, L. Petrosanti, S.K. Nematov, T.N. Chernikova, K. Thor and J. Leipner (2008). Response of the photosynthetic apparatus of cotton (Gossypium hirsutum L.) to the onset of drought stress under field conditions studied by gas-exchange analysis and chlorophyll fluorescence imaging. Plant Physiol. Biochem. 46:189-195.

McMichael, B.L. and J.D. Hesketh (1982). Field investigations of the response of cotton to water deficits. Field Crops Res., 5: 319- 333.

Megha, B. R., U. V. Mummigatti, V.P. Chimmad and Y. R. Aladakatti (2017). Evaluation of Hirsutum Cotton Genotypes for Water Stress using Peg-6000 by Slanting Glass Plate Technique Int. J. Pure App. Biosci. 5 (2): 740-750.

Mert, M. (2005). Irrigation of cotton cultivars improves seed cotton yield, yield components and fibre properties in the Hatay region, Turkey. Acta Agronomy Scand. 55: 44-50.

Michel, B.E. and M.R. Kaufmann (1973). The Osmotic Potential of Polyethylene Glycol 60001. Plant Physiol., 51: 914916.

Moinuddin, Fischer R. A., K.D. Sayre and M. P. Reynolds (2005). Osmotic Adjustment in wheat in relation to grain yield under water deficit. Environments Agronomy. Journal 97, 1062- 1071. 
Murillo-Amador, B., R. Lopez-Aguilar, C. Kaya, J. Larrinaga-Mayoral and A. Flores-Hernandez (2002). Comparative effect of $\mathrm{NaCl}$ and $\mathrm{PEG}$ on germination emergence and seedling growth of cowpea. J. Agron. Crop Sci., 188: 235247.

Osborne, S. and J.C. Banks (2006). The effects of water stress during bloom on lint yield, fiber quality and price. Beltwide Cotton Conferences, San Antonio, Texas, January, 3-6: 16791780.

Pettigrew, W.T. (2004a). Physiological consequences of moisture deficit stress in cotton. Crop Sci., 44: 12651272.

Pettigrew, W.T. (2004b). Moisture deficit effects on cotton lint yield, yield components, and boll distribution. Agronomy J. 96: 377-383.

Pettigrew, W.T. and W.R. Meredith (1994). Leaf gas exchange parameters vary among cotton genotypes. Crop Sci., 34:700-705.

Pirdashti, H., Z. Sarvestani Tahmasebi, G.H. Nematzadeh and A. Ismail (2003). Effect of water stress on seed germination and seedling growth of rice (Oryza sativa L.) genotypes. Pakistan J. of Agronomy 2: 217-222.

Quisenberry, J.E., B. Roark, D.W. Fryer and R.J. Kohel (1980). Effectiveness of selection in upland cotton in stress environments. Crop Sci., 20: 450-453.

Quisenberry, J.E., W.R. Jordan, B.A. Roark and D.W. Fryrear (1981). Exotic cottons as genetic sources for drought resistance. Crop Sci., 21: 889895.

Rashid, A., J.C. Stark, A. Tanveer and T. Mustafa (1999). Use of canopy temperature measurements as a screening tool for drought tolerance in spring wheat. J. Agron. Crop Sci., 182: 231-237.

Ritchie, G.L., C.W. Bednarz, P.H. Jost and S.M. Brown (2004). Cotton Growth and Development. Bulletin 1252. Cooperative Extension Service and the University of Georgia College of
Agricultural and Environmental Sciences, Athens, GA, USA.

Sezener, V., H. Basal, C. Peynircioglu, T. Gurbuz and K. Kizilkaya (2015). Screening of cotton cultivars for drought tolerance under field conditions. Turk j field crops, 20(2), 223-232.

Sinclair, T.R. (2005). Theoretical analysis of soil and plant traits influencing daily plant water flux on drying soils. Agronomy Journal. 97: 1148-1152.

SPSS (1995). SPSS Computer User's Guide SPSS in USA.

Steel, R.G.D. and J.H. Torrie (1960). Principles and Procedures of Statistics. McGraw-Hill Book Company Inc., New York. USA.

Tonin, G. A., N. M. Carvalho, S. N. Kronka and A. S. Ferraudo (2000). Culture systems, velvet bean and mineral fertilization influence on maize seeds physiological quality. Revista Brasileira de Sementes, 22: 276-279.

Turner, N.C. (1997). Further progress in crop water relations. Adv. Agron. 58:293-338.

Turner, N.C., A.B. Hearn, J.E. Begg and G.A. Constable (1986). Cotton (Gossypium hirsutum L.) physiological and morphological responses to water deficits and their relationship to yield. Field Crops Res. 14:153-170.

Ullah, I.M. Rahman and Y. Zafar (2006). Genotypic variation for drought tolerance in cotton (Gossypium hirsutum L.) Seed cotton yield responses. Pak. J. Bot. 38: 1679-1687.

Voltas, J., H. Lopez-Corcoles and G. Borras (2005). Use of biplot analysis and factorial regression for the investigation of superior genotypes in multi environment trials. Eur. J. Agronomy. 22: 309-324.

Wang, C., A. Isoda and P. Wang (2004). Growth and yield performance of some cotton cultivars in Xinjiang, China, an arid area with short growing period. J. Agronomy Crop Sci., 190: 177-183.

Zhu, J.K. (2001). Plant salt tolerance. Trends Plant Sci., 6: 66-71. 
تقييم التباين الوراثي لبعض التراكيب الوراثية لقطن البربادنس للإجهاد المائي

محمد أحمد عاثور الدهان ، عرفة بلرى عبدالكريم الفشيقاوى ، يسري ابراهيم محمد الحبيني

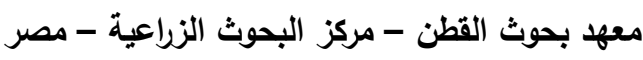

الملخص العربي

يهذف هذا البحث إلي تقدير تباين التراكيب الوراثيه في قطن الباربادينس للاستجابة لظروف الإجهاد المائي و

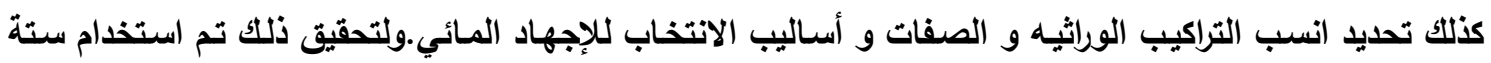
عثر تركيب وراثي تثكل مدى واسع من الخصائص المحصوليه و الغزليه في تجريتين متلازمتين (معطليه و حقليه)

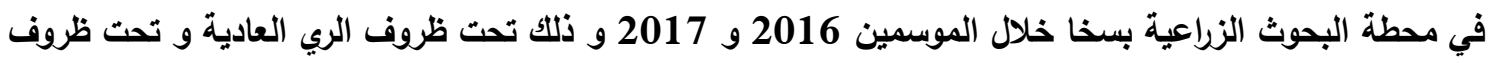
الاجهاد المائي ـ بالنسبه للتجريه المعطلية تم محاكاة الاجهاد المائي عن طريق استخدام البولي ايثليلين جليكول 600 لإستحثاث ضفط اسموزي يقترب من الصفر و -0.4 ميجا باسكال لمحاكاة الظروف العادية و ظروف الاجهاد

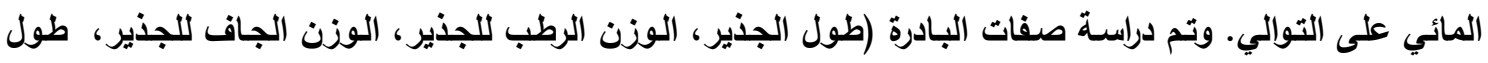
المجموع الخضري، الوزن الرطب للمجموع الخضري و الوزن الجاف للمجموع الخضري) . و بالنسبه للتجريه الحقليه فتم قياس صفات المحصول و مكوناته و بعض الصفات الخضرية و بعض صفات التيلة و قد أظهرت النتائج ما

1. أظهر تحليل التباين لموسمي الدراسه 2016 و 2017 وجود فروق معنويه لأنظمة العياه و للتراكيب الوراثيه و التفاعل بين هين العاملين لمعظم الصفات العدروسة مما يؤكد تباين التراكيب الوراثيه تحت الظروف المائيه

المختلفه.

2. أظهرت قيم المتوسطات و جود انخفاض لكل الصفات المدروسه نتيجه للإجهاد المائي مقارنة بالظروف

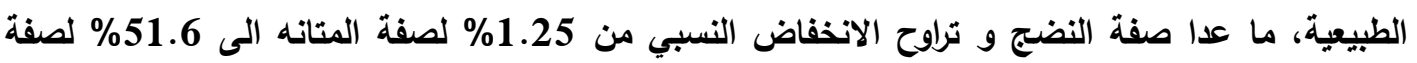
مصصول القطن الثعر للنبات. كما تلاحظ أن صفات التيلة كانت اقل الصفات تأثرا بالإجهاد المائي. 3. بالنسبة لصفات البادرة اظهرت التراكيب الوراثيه جيزة 88 و جيزة 93 و الاشموني و سوفين تحملا للاجههاد

العائي. 4. بالنسبه للصفات الخضريه اظهرت التراكيب الوراثيه جيزة 77 ، جيزة 94 ، جيزة 89 جيزة 86 ، الأشموني ، المنوفي والسوفين تحملا تحت ظروف الإجهاد المائي.

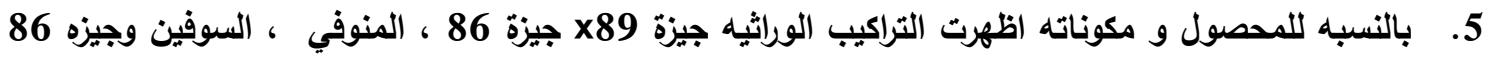

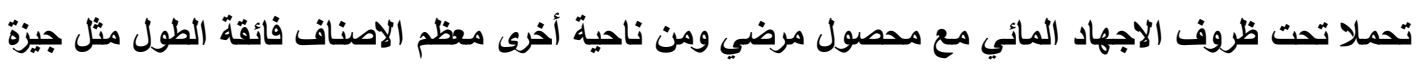
87 ، جيزة 88 و جيزة 93 كانت من اكثر الاصناف حساسية للإجهاد المائي من وجهة النظر المحصوليه. 
6. اظهر معامل حساسية الجفاف (DSI) ارتباط معنوي سالب مع المحصول تحت ظروف الاجهاد المائي مما يؤكد امكانية استخدام هذا المعامل لتحديد الاصناف المتحمله للإجهاد المائي وكذلك ضرورة اجراء تقييم التراكيب الوراثيه تحت ظروف الاجهاد عند التربيه لتحمل الاجهاد المائي. 7. أظهرت معظم الأصناف فائقة الطول حساسية أكثر للإجهاد المائي بالنسبة لصفات التيلة. 8. اظهر معامل الارتباط قيما موجبة ومعنوية بين محصول القطن الزهر للنبات وكل من معظم الصفات المحصوليه و ارتفاع النبات و كان الارتباط سالب مع معظم صفات التيلة. 9. اظهر تحليل معامل المرور ان اعلى تأثير مباثر على صفة محصول القطن الزهر كانت لصفات عدد اللوز على النبات (1.362) و يتبعها وزن اللوزه (0.98) ثم معدل الحليج (0.534) و اعلى تأثير غير مباشر لمعظم صفات المحصول و الصفات الخضريه كانت من خلال صفتي عدد اللوز على النبات و وزن اللوزه.مما يؤكد ان الإنتخاب لتحسين الإنتاجيه تحت ظروف الإجهاد المائي قد تكون اكثر فاعليه خلال الاتتخاب المباشر لصفتى عدد اللوز / النبات و وزن اللوزه. 10. اكد التحليل العاملي على اهمية الصفات المحصوليه و ارتفاع النبات لتحسين الإنتاجية تحت ظروف الإجهاد المائي. 
Evaluation of the genetic variation for some genotypes in cotton 